Draft Version OCTOBER 31, 2018

Preprint typeset using IATEX style emulateapj v. 14/09/00

\title{
RADIO EMISSION AND THE TIMING PROPERTIES OF THE HARD X-RAY STATE OF GRS $1915+105$
}

\author{
Michael P. Muno, Ronald A. Remillard, Edward H. Morgan, \\ Department of Physics and Center for Space Research, Massachusetts Institute of Technology, 77 \\ Massachusetts Ave., Cambridge, MA 02139 \\ muno@mit.edu,ehm@space.mit.edu,rr@space.mit.edu \\ ElizaBeth B. WALtMan \\ Remote Sensing Division, Naval Research Laboratory, Code 7210, Washington, DC 20375 \\ ewaltman@rsd.nrl.navy.mil \\ Vivek Dhawan, Robert M. HJellming \\ National Radio Astronomy Observatory, Socorro, NM 87801 \\ vdhawan@aoc.nrao.edu \\ Guy PoOley \\ Mullard Radio Astronomy Observatory, Cavendish Laboratory, Madingley Road, Cambridge CB3 0HE \\ ggp1@cam.ac.uk \\ Draft version October 31, 2018
}

\begin{abstract}
We combine a complete sample of 113 pointed observations taken with the Rossi X-ray Timing Explorer between 1996-1999, monitoring observations taken with the Ryle telescope and the Green Bank Interferometer, and selected observations with the Very Large Array to study the radio and X-ray properties of GRS 1915+105 when its X-ray emission is hard and steady. We establish that radio emission always accompanies the hard-steady state of GRS $1915+105$, but that the radio flux density at $15.2 \mathrm{GHz}$ and the X-ray flux between 2-200 keV are not correlated. Therefore we study the X-ray spectral and timing properties of GRS 1915+105 using three approaches: first, by describing in detail the properties of three characteristic observations, then by displaying the time evolution of the timing properties during periods of both faint and bright radio emission, and lastly by plotting the timing properties as a function of the the radio flux density. We find that as the radio emission becomes brighter and more optically thick, 1) the frequency of a ubiquitous $0.5-10 \mathrm{~Hz}$ QPO decreases, 2) the Fourier phase lags between hard $(11.5-60 \mathrm{keV})$ and soft $(2-4.3 \mathrm{keV})$ in the frequency range of $0.01-10 \mathrm{~Hz}$ change sign from negative to positive, 3) the coherence between hard and soft photons at low frequencies decreases, and 4) the relative amount of low frequency power in hard photons compared to soft photons decreases. We discuss how these results reflect upon basic models from the literature describing the accretion flow around black holes and the possible connection between Comptonizing electrons and compact radio jets.
\end{abstract}

Subject headings: black hole physics - stars: individual (GRS 1915+105) - X-rays: stars

\section{INTRODUCTION}

The microquasar GRS $1915+105$ is one of the most interesting galactic sources of radio, infrared, and X-ray emission. It was first discovered as a transient X-ray source by Granat in 1992 (Castro-Tirado et al. 1992), and later was observed to emit highly relativistic radio jets with intrinsic velocities greater than $0.9 \mathrm{c}$ (Mirabel \& Rodríguez 1994; Fender et al. 1999b). High optical extinction has prevented studies of the binary companion of GRS 1915+105, and there is no known orbital period or mass function for the system. However, the X-ray luminosity of GRS $1915+105$ greatly exceeds the Eddington luminosity for a neutron star (Greiner, Morgan, and Remillard 1996), and the other galactic sources of highly relativistic (> 0.9c) jets, GRO J1655-40 (Orosz \& Bailyn 1997; Shahbaz et al. 2000) and V4641 Sgr (Orosz et al. 2001), have mass functions greater than the maximum mass for a neutron star, $3 \mathrm{M}_{\odot}$. GRS $1915+105$ is therefore thought to be a black hole binary system. The properties of microquasars in the galaxy are reviewed by Mirabel \& Rodríguez (1999).

Although GRS $1915+105$ is often noted for exhibiting X- ray, radio, and infrared emission which is variable on time scales of seconds to minutes (Greiner et al. 1996; Pooley \& Fender 1997; Mirabel et al. 1998; Eikenberry et al. 1998), the source also exhibits steady emission which is reminiscent of black hole candidates such as $\mathrm{Cyg} \mathrm{X}-1$ and GX 339-4. Both the steady and variable emission from GRS 1915+105 exhibit two (Muno, Morgan, \& Remillard 1999; Rao, Yadav, \& Paul 2000) or three (Belloni et al. 2000) basic modes of X-ray emission. In the two-state description, the hard state resembles the very high state of canonical black hole binaries (Morgan, Remillard, \& Greiner 1997), as it exhibits X-ray flux with a power-law spectrum above $40 \mathrm{keV}$, prominent thermal emission from the accretion disk below $\sim 5 \mathrm{keV}$, and a strong (up to $15 \%$ RMS) $0.5-10 \mathrm{~Hz}$ quasi-periodic oscillation (QPO). Conversely, the soft state of GRS 1915+105 exhibits little X-ray emission above $40 \mathrm{keV}$ and no $0.5-10 \mathrm{~Hz}$ QPO, although a stationary $67 \mathrm{~Hz} \mathrm{QPO}$ and several low frequency $(<0.1 \mathrm{~Hz})$ QPOs are often observed during this state.

Long-term radio and X-ray monitoring of GRS 1915+105 (Rodríguez et al. 1995; Foster et al. 1996; Harmon et al. 1997; Pooley \& Fender 1997; Bandyopadhyay et al. 1998) has revealed that periods of hard, 
Muno et al.

steady X-ray emission are often accompanied by bright, optically thick radio emission. This has been dubbed the "plateau state" by Foster et al. (1996; see also Fender 2001). The radio plateau emission in GRS 1915+105 resembles steady, optically thick radio emission observed coincident with hard X-ray emission in the black hole candidates Cyg X-1 (Hjellming, Gibson, \& Owen 1975; Brocksopp et al. 1999), GX 339-4 (Corbel et al. 2000), and Cyg X-3 (Waltman et al. 1995; McCollough et al. 1999). The connection between the X-ray and radio emission in GRS 1915+105 is illustrated in Figure 1, where we plot the intensity as a function of time as observed in soft X-rays by the All-Sky Monitor (ASM) aboard the Rossi X-ray Timing Explorer (RXTE; first panel), and at radio wavelengths by the Ryle telescope $(15.2 \mathrm{GHz}$; third panel). We also plot the the ASM hardness ratio HR2 (5-12 keV / 3-5 keV; second panel), and the radio spectral index $\alpha$ taken from monitoring data at 2.25 and $8.3 \mathrm{GHz}$ from the Green Bank Interferometer (i.e. $S_{\nu} \propto \nu^{\alpha}$; only observations where $S_{\nu}$ at both wavelengths are greater than $20 \mathrm{mJy}$ are plotted). Bright radio plateau emission and hard-steady X-ray emission is evident on several occasions (e.g. MJD 50730-50750), yet the radio emission is sometimes a factor of 10 fainter during other hard $\mathrm{X}$ ray states (e.g. MJD 50450-50550). Observations with the Very Long Baseline Array during the plateau state reveal that the optically thick radio emission originates in a compact jet on order 10 AU across (Dhawan, Mirabel, \& Rodríguez 2000).

The radio plateau state is of further interest because it is often preceded and/or followed by optically thin radio flares in GRS 1915+105 (Fender et al. 1999b), as are similar hard-steady states with optically thick radio emission in GX 339-4 (Corbel et al. 2000) and Cyg X-3 (McCollough et al. 1999). The optically thin emission in GRS 1915+105 originates from relativistic ejections of synchrotron-emitting blobs detached from the central source (Mirabel \& Rodríguez 1994; Fender et al. 1999b; Dhawan et al. 2000). In Figure 1, optically thin radio flares precede and terminate the radio plateau emission which occurred between MJD 50730-50750, while weaker flares with shorter duration terminate the long period of weaker radio emission from MJD 50450-50550. These events are associated with flares in the ASM light curve, which suggests that the most powerful jets are associated with instabilities in the inner accretion disk. Moreover, there have been several occasions in which weaker radio and infrared flares recur at $\sim 30$ minute intervals, and are preceded by distinctive dip and flare patterns in X-rays (Pooley \& Fender 1997; Eikenberry et al. 1998; Mirabel et al. 1998).

During the plateau state of GRS $1915+105$, the radio luminosity is low compared to the large optically thin flares, but the power in the radio jets may still represent a significant fraction of the accretion energy inferred from the X-ray emission (Falcke \& Biermann 1999; Fender 2001). Since this emission originates from a compact jet within $10 \mathrm{AU}$ of the central source and is present only during the hard X-ray state, it seems very likely that the compact jet is influenced by the properties of the inner accretion flow, and vice versa. This has led Fender et al. (1999a) to suggest that the hard $\mathrm{X}$-rays originate from inverse Compton scattering of seed photons at the base of the compact jet of relativistic electrons. This idea motivates further efforts to compare the properties of the radio emission to the $\mathrm{X}$ ray spectral and timing information during the hard state of GRS $1915+105$.

The timing properties of GRS $1915+105$ provide a promising means of probing the accretion flow near the black hole. In particular, the $0.5-10 \mathrm{~Hz}$ QPO observed during the hard state appears to provide a link between the optically thick accretion disk and the Comptonizing electrons, because (1) the frequency of the QPO increases as the thermal flux from the disk increases (Chen, Taam \& Swank 1997; Trudolyubov, Churazov, \& Gilfanov 1999; Markwardt, Swank, \& Taam 1999), (2) the spectrum of the QPO amplitude is hard, indicating an origin in the power law component of the spectrum (Morgan et al. 1997), and (3) the QPO is only present when the power-law component of the X-ray spectrum is strong, while it is absent when the power-law component is weak (Muno et al. 1999). Additional timing characteristics, such as the Fourier phase lags and the coherence function, may further constrain the relationship between hard and soft Xray components. Reig et al. (2000) have found that the signs of the phase lags change from positive to negative as the frequency of the $0.5-10 \mathrm{~Hz}$ QPO increases, which has provided impetus for others (e.g. Nobili et al. 2000) to develop models describing the structure of the Comptonizing corona and how it is related to the optically thick accretion disk.

In this paper, we use a complete sample of "hardsteady" observations of GRS 1915+105 (referred to as the $\chi$ state by Belloni et al. 2000) to examine in detail the connection between the hard, steady X-ray emission and the steady radio emission. First, we establish that the hardsteady X-ray states of GRS $1915+105$ always exhibit detectable radio emission. However, the intensities of the 2$200 \mathrm{keV} \mathrm{X}$-ray and $15.2 \mathrm{GHz}$ radio flux are not correlated, so we proceed to examine whether there are more subtle relationships between the spectrum and timing properties of the X-ray emission and the intensity and spectrum of the radio emission. We investigate these correlations in three different manners: first, by describing in detail the properties of three characteristic observations, then by displaying the time evolution of the timing properties during periods of both faint and bright radio emission, and lastly by examining the timing properties as a function of the radio flux density.

\section{OBSERVATIONS AND DATA ANALYSIS}

\subsection{Ryle Monitoring Observations}

The Ryle Telescope at the Mullard Radio Astronomy Observatory, Cambridge, is primarily used for microwavebackground measurements, but during gaps in that program it has been used to monitor variable galactic and extra-galactic sources. It operates at $15.2 \mathrm{GHz}$, and uses a single set of linearly-polarized feeds. In good observing conditions, the RMS noise in a 1 minute integration is about $3.5 \mathrm{mJy}$. Since the measurements are unbiased, the noise level decreases as the square root of the observing time. Details of the observing and analysis routine are given in Pooley \& Fender (1997).

We have averaged the radio measurements in two ways 
for the purposes of this paper. We have used the averaged daily flux density measured with the Ryle Telescope when displaying monitoring light curves of GRS 1915+105 (e.g. Figure 1). When comparing the Ryle measurements to the properties of pointed $R X T E$ observations, we have averaged data taken within 0.5 days before and after the $R X T E$ observation in order to sample the radio properties which most closely correspond to the X-ray observation (see Table 1).

\subsection{Green Bank Interferometer Monitoring Observations}

Monitoring with the Green Bank Interferometer has been performed simultaneously at 2.25 and $8.3 \mathrm{GHz}$ on a $2.4 \mathrm{~km}$ baseline with $35 \mathrm{MHz}$ bandwidth and dual circular polarization. Most scans are 10-15 minute integrations, and the calibration procedure is described by Foster et al. (1996). Random (one sigma) errors in the GBI data are flux dependent: $4 \mathrm{mJy}(2 \mathrm{GHz})$ or $6 \mathrm{mJy}(8 \mathrm{GHz})$ for fluxes $<100 \mathrm{mJy}, 15 \mathrm{mJy}(2 \mathrm{GHz})$ or $50 \mathrm{mJy}(8 \mathrm{GHz})$ for fluxes $\simeq 1 \mathrm{Jy}$. In addition, systematic errors are introduced by atmospheric and hardware effects, which we estimate may approach $10 \%$ at $2 \mathrm{GHz}$ and $20 \%$ at $8 \mathrm{GHz}$, occasionally higher for extreme local hour angles. Flux density measurements below $20 \mathrm{mJy}$ approach the noise level of the instruments, and therefore we do not include measurements below this level in our analysis.

As with the Ryle data, daily GBI measurements have been averaged when producing monitoring light curves, and GBI data taken within 0.5 days of a pointed $R X T E$ observation have been averaged when comparing individual observations (Table 1). In both cases, we also have computed the spectral index $\alpha$ from the average flux in the 2.25 and $8.3 \mathrm{GHz}$ bands.

\subsection{Very Large Array Observations}

The VLA is a multi-frequency, multi-configuration aperture synthesis imaging instrument, consisting of 27 antennas of $25 \mathrm{~m}$ diameter. The receivers at $1.42,5.0,8.45$, and 15.2 GHz, have been used on various occasions, with 2 adjacent bands of $50 \mathrm{MHz}$ nominal bandwidth processed in continuum mode. The corresponding 1-sigma sensitivities in 10 minutes are $0.06,0.06,0.05$, and $0.2 \mathrm{mJy}$ respectively. Switching between multiple frequencies takes about $30 \mathrm{~s}$. The array configuration is varied every 4 months to cycle between 4 sets, A, B, C, and D, with maximum baselines of about $36,11,3.4$, and $1 \mathrm{~km}$.

Observations of GRS $1915+105$ while in its radio faint state have been obtained from the public archive in order to constrain the radio spectrum. The observations we have found are listed in Table 2, along with mean fluxes from Ryle and GBI observations taken within a half day of the VLA observations. For all of the observations reported here, GRS $1915+105$ was unresolved by the synthesized beam (see Table 2) in any configuration at any wavelength. Other sources in the field of view (mainly at $1.4 \mathrm{GHz}$ ) are well separated in the images, so there is no confusion with GRS $1915+105$.

Calibration and imaging were carried out with standard tasks in the NRAO AIPS (Astronomical Image Processing System) package. For all of these observations, the phase calibrator was $1925+211$. The primary flux density calibrator was 3C286 $(1328+307)$ for all observations except 1996 November 14, when 3C48 $(0134+329)$ was used. In practice, the flux density errors are not set by the RMS receiver (thermal) noise stated above, but by errors in the flux density scale, estimated to be $3-5 \%$ of the measurement, and/or source variability, depending on the occasion. More details of the VLA are given in the Observational Status Summary (http://www.aoc.nrao.edu/vla/obstatus/vlas/vlas.html), and the data reduction is treated in formal detail in Taylor, Carilli, \& Perley (1999) and in the VLA cookbook (http://www.cv.nrao.edu/aips/cook.html).

\subsection{Pointed RXTE Observations}

The Rossi X-ray Timing Explorer (RXTE) consists of three instruments: the All-Sky Monitor (ASM; Levine et al. 1996), the Proportional Counter Array (PCA; Jahoda et al. 1996), and the High Energy Timing Experiment (HEXTE; Rothschild et al. 1998). Figure 1 shows the ASM light curve of GRS $1915+105$ along with daily radio measurements from Ryle and the spectral index from the GBI. There is no apparent correlation between the radio emission and the ASM count rate, although there is some correlation with the ASM hardness ratio HR2 (5-12 keV / $3-5 \mathrm{keV})$. In addition, there has been a long campaign of weekly observations with with the PCA and HEXTE, which we use to study the detailed timing and spectral properties of the hard emission from GRS 1915+105. We define intervals as "hard-steady" if the ratio of the mean PCA count rates in the $12-60 \mathrm{keV}$ to the $2-12 \mathrm{keV}$ bands exceeds 0.05 and the mean variability in the count rate $(2-$ $60 \mathrm{keV}$ ) at 1s time intervals is less than $12 \%$ for an $R X T E$ orbit (typically $3000 \mathrm{~s}$ ). In Figure 1 we have marked the pointed RXTE observations of the hard-steady state with vertical bars above the ASM light curve, and we have listed them in Table 1. The small dots represent the remainder of the pointed observations. We have analyzed all $113 \mathrm{ob}-$ servations of GRS $1915+105$ in the hard steady state taken with $R X T E$ between 1996 April 15 (MJD 50188) through 1999 March 22 (MJD 51259), during the time interval for the PCA instrument gain setting known as "epoch 3". In order to improve the signal-to-noise of the spectral and timing measurements whenever possible, if the variability in the mean count rate in $16 \mathrm{~s}$ bins is less than $5 \%$ during a single day, we have analyzed all of the data for that day together. Otherwise, we have grouped the data while remaining within the $5 \%$ variability limit (16 s bins), with the smallest unit being a single $R X T E$ orbit.

For each time unit, we have integrated 128-channel energy spectra ("Standard2") data from PCUs 0 and 1 of the PCA, and 64 channel (archive mode) spectra from both clusters of HEXTE. In order to calculate the flux from GRS $1915+105$, we have fit the spectra with the phenomenological model described in Muno et al. (1999). The model consists of the sum of a multi-color blackbody, a power law (with a high-energy cutoff when necessary), and a Gaussian line (representing iron emission between 5-7 keV). Interstellar absorption is taken into account assuming a column density equivalent to $6 \times 10^{22} \mathrm{~cm}^{2}$. A normalization factor is allowed to account for differences in the effective area of the PCUs and the HEXTE clusters which are not taken into account by the response matrices. The standard FTOOLS 4.2 background sub- 
Muno et al.

traction algorithm for bright sources has been applied to the data, and a systematic uncertainty of $1 \%$ has been added to each spectral bin. We have computed an X-ray flux for each observation, by summing the bolometric flux implied by multi-temperature disk model $\left(F_{b b}=1.08 \times\right.$ $10^{-11} N_{\text {app }} \sigma T_{\text {app }}^{4}$ ), the flux in the power law between 2-200 $\operatorname{keV}\left(\int_{2}^{200} 1.60 \times 10^{-9} N_{\Gamma} E^{-\Gamma+1} \exp \left[-E / E_{\text {cut }}\right] d E\right)$, and the flux in the iron line $\left(N_{\text {Gauss }} /\left(\sqrt{2 \pi} W_{\text {Gauss }}\right) \int_{0}^{\infty} \exp [0.5(E-\right.$ $\left.\left.\left.E_{\text {Gauss }}\right)^{2} / W_{\text {Gauss }}^{2}\right] d E\right)$.

We also have created power density spectra (PDS) and cross spectra (CS) for each $256 \mathrm{~s}$ interval of data with $2^{-7}$ $\mathrm{s}$ time resolution and four energy channels, using combinations of the binned and event modes for each observation. The four energy channels used are as follows: $2-4.3 \mathrm{keV}$ (henceforth referred to as the "low energy" band), 4.3-7.8 $\mathrm{keV}, 7.8-11.5 \mathrm{keV}$, and $11.5-60 \mathrm{keV}$ (referred to as the "high energy" band). The PDS and CS have been averaged for the entire time unit (an $R X T E$ orbit or an observation, as defined above) and logarithmically re-binned. The PDS have been corrected for dead-time effects and Poisson noise (Morgan et al. 1997), and have been normalized to the fractional RMS squared per Hz. To quantify the features of the PDS, we have fit PDS from each energy band with a model consisting of a power law with two breaks and Lorentzians for any QPOs present in the PDS. We have searched for the $67 \mathrm{~Hz}$ QPO during each of these hard-steady observations using data with $2^{-13} \mathrm{~s}$ time resolution in a single energy channel, but we did not detect it. Typical upper limits range from $0.1-0.5 \%(1 \sigma)$.

The coherence function and phase lags have been calculated from the CS in the manner described in Vaughan \& Nowak (1997). We have used the standard convention that "hard" or "positive" lags indicate that higher energy photons lag behind lower energy photons. The uncertainties have been calculated for the case of high signal power and high measured coherence, using an estimate of the dead-time-corrected Poisson noise (Morgan et al. 1997). If the signal power is smaller than the estimated noise, or if the coherence is less than the noise divided by the signal, we do not plot either the phase lags or the coherence functions.

Guided by the long-term monitoring light curves in Figure 1, we have defined subsets of the hard-steady observations based upon the radio emission from GRS $1915+105$ in order to facilitate the comparison between the radio and X-ray emission. We have defined "radio plateau" hard-steady conditions for observations for which GRS $1915+105$ is brighter than $20 \mathrm{mJy}$ at $15.2 \mathrm{GHz}$, and exhibits a flat radio spectrum with index (measured from the daily average of the flux) $\alpha>-0.2$. We also have defined "radio steep" conditions for observations when GRS $1915+105$ is brighter than $20 \mathrm{mJy}$ at $15.2 \mathrm{GHz}$, and exhibits a optically thin radio spectrum with index $\alpha<-0.2$. Figure 1 demonstrates that the radio steep conditions typically represent the transition into and out of periods of radio plateau emission, and occur during the decays of large optically thin radio flares (see also Fender et al. 1999b; Dhawan et al. 2000). Our discussion of radio steep observations will be limited, as the optically thin emission probably results from radio ejecta that could evolve independently of the instantaneous X-ray conditions on 0.5 day time scales.
Finally, we have defined "radio-faint" conditions for those hard-steady X-ray observations for which the daily average of the radio flux at $15.2 \mathrm{GHz}$ is less than $20 \mathrm{mJy}$, because for these observations the radio flux at the GBI frequencies is too low to reliably measure a spectral index. The radio faint conditions occur with two variantsthose observations for which the X-ray emission is bright, and those for which it is faint. The behavior of the timing properties with respect to the radio emission is similar for both variants, although the shape of the X-ray spectrum and the values of the timing properties (e.g. the QPO frequency and the phase lags) are distinctive, and warrant a separate discussion.

\section{RESULTS}

We first investigate 1) whether all of the hard-steady observations are coincident with radio emission, 2) whether the X-ray and radio flux are correlated, and 3) how the radio conditions (plateau, steep, and faint) are distributed in a plot of radio vs. X-ray flux. Figure 2 displays a plot of the radio flux density at $15.2 \mathrm{GHz}$ (log scale) versus the $2-200 \mathrm{keV}$ X-ray flux for each of the hard-steady observations. It is clear that all hard steady observations have radio flux densities greater than $2 \mathrm{mJy}$. Panel 3 of Figure 1 shows that the radio flux from GRS $1915+105$ does drop below 1 mJy (e.g. MJD 50825-50875), but such intervals are associated with the soft X-ray state. Thus, radio emission always accompanies the hard-steady X-ray state, although a note of caution is appropriate for those observations associated with variable, optically thin radio emission, as noted above. However, Figure 2 also shows the radio and X-ray fluxes are not correlated in the hardsteady state. Likewise, we have found that the total 50-100 keV flux, the power law flux, and the thermal (multi-color disk) flux are not correlated with the strength of the radio emission (not shown).

The radio conditions are represented in Figure 2 as follows: radio plateau observations are plotted as triangles, radio steep as circles, and radio faint as $\times$ 's. Observations for which the GBI spectral index has not been measured are plotted only with error bars. The plot can be roughly split into four quadrants. The radio plateau observations are clearly localized in the upper left quadrant, with $S_{15.2}>20 \mathrm{mJy}$ and $F_{\mathrm{X}}<4 \times 10^{-8} \mathrm{erg} \mathrm{cm}^{-2}$ $\mathrm{s}^{-1}$. Some radio steep observations are also present in this quadrant. The upper right quadrant contains radio steep observations and observations for which no spectral index is available from the GBI. These latter observations were taken between MJD 50313-50320 (before the current GBI monitoring program), a time interval which is included in the analysis of Bandyopadhyay et al. (1998). During this time span a large radio flare occurred terminating a plateau state, and the radio flux density at $1.4 \mathrm{GHz}$ was consistently larger than that at $3.3 \mathrm{GHz}$, which suggests that these points should be classified as radio steep. Therefore, the upper right quadrant $\left(S_{15.2}>20 \mathrm{mJy}\right.$ and $\left.F_{\mathrm{X}}>4 \times 10^{-8} \mathrm{erg} \mathrm{cm}^{-2} \mathrm{~s}^{-1}\right)$ appears to include only radio steep observations, during which the radio and $\mathrm{X}$-ray emission may vary independently since the radio measurements may be dominated by the evolution of an expanding jet detached from the central source. For this reason, we choose to ignore the upper right quadrant. 
The lower quadrants, with $S_{15.2}<20 \mathrm{mJy}$, contain radio faint observations by construction. After examining the temporal clustering of these radio-faint observations, we feel that it is useful also to consider them in two quadrants: an X-ray faint quadrant with $F_{\mathrm{X}}<4 \times 10^{-8} \mathrm{erg} \mathrm{cm}^{-2}$ $\mathrm{s}^{-1}$, and an X-ray bright quadrant with $F_{\mathrm{X}}>4 \times 10^{-8} \mathrm{erg}$ $\mathrm{cm}^{-2} \mathrm{~s}^{-1}$. This separation is further motivated by their differing energy spectral and timing properties, which are described in the next section.

\subsection{Characteristic Observations}

From each of the three quadrants under consideration from the radio versus X-ray flux plane (Figure 2), we have selected a characteristic observation in order to illustrate the range of $\mathrm{X}$-ray spectral and timing properties within the hard-steady X-ray state. In Figures 3, 4, and 5, the left panel is a radio faint, X-ray faint observation (MJD 50488; 1997 February 9); the center panel is a radio plateau, Xray faint observation (MJD 50737; 1997 October 16); and the right panel is a radio faint, X-ray bright observation (MJD 50708; 1997 September 17). The radio and X-ray fluxes from these three observations are indicated in Figure 2 .

\subsubsection{X-ray Energy Spectra}

Figure 3 illustrates the results of the spectral fits for the three representative observations with differing values of the radio and X-ray flux. The spectrum in the left panel is from a radio faint observation when the $\mathrm{X}$-ray emission was also relatively faint $\left(2.2 \times 10^{-8} \mathrm{erg} \mathrm{cm}^{-2} \mathrm{~s}^{-1}\right)$. The soft emission during this observation can be modeled $\left(\chi_{\nu}^{2} \sim 1\right)$ using a cool multi-temperature blackbody with an apparent inner disk temperature of $T_{\text {app }} \simeq 0.8 \mathrm{keV}$ and an apparent radius of $R_{\text {app }} \simeq 40-80 \mathrm{~km}$ (assuming a distance of $11 \mathrm{kpc}$ and an inclination angle of $66^{\circ}$; see Fender et al. 1999b). The power law has a photon index $\Gamma \simeq 2$ and requires an exponential cutoff at about $70 \mathrm{keV}$. The results of this spectral fit are characteristic of observations during which both the X-ray and radio flux were low.

The spectrum in the middle panel is from an observation which exhibited radio plateau conditions when the X-ray emission was also faint $\left(2.6 \times 10^{-8} \mathrm{erg} \mathrm{cm}^{-2} \mathrm{~s}^{-1}\right)$. Despite the similarity in X-ray flux to the left panel, the shape of the X-ray spectrum is quite different. The thermal component of the model appears hot $\left(T_{\text {app }}>3 \mathrm{keV}\right)$ with a small apparent inner radius $\left(R_{\text {app }}<5 \mathrm{~km}\right)$. This quasi-thermal component is not consistent with simple models of reflection from an accretion disk (e.g. Magdziarz \& Zdziarski 1995). It is possible that this broad thermal bump represents emission from a relatively small area of the disk which has been heated by, for example, a magnetic flare (di Matteo, Celotti, \& Fabian 1999) or a spiral shock wave (Tagger \& Pellat 1999), or that the disk develops an optically thick atmosphere which Comptonizes the emergent spectrum, so that the spectral hardening and decreased apparent radius are illusory effects of increased scattering in a "puffed up" disk (Merloni, Fabian, \& Ross 2000). The photon index of the power law is $\Gamma \simeq 2.7$, and a cut-off at about $80 \mathrm{keV}$ is required for observations with longer integration times and hence better statistics at energies above $100 \mathrm{keV}$. We note that Rao et al. (2000a) have presented a different model for spectra of GRS $1915+105$ such as these, consisting of a multi-temperature disk, a thermalCompton spectrum (Sunyaev \& Titarchuk 1980), and a power law. For the purposes of this paper, we prefer to use our simpler model with fewer free parameters.

The right panel is from an observation when the radio emission was faint $(6 \mathrm{mJy})$ and the X-ray emission was bright $\left(8.4 \times 10^{-8} \mathrm{erg} \mathrm{cm}^{-2} \mathrm{~s}^{-1}\right)$. The spectrum is similar to the radio bright, $\mathrm{X}$-ray faint observation in the center panel, except that the relative contribution of the power law flux increases dramatically. The thermal component is hot $\left(T_{\text {app }}>2.5 \mathrm{keV}\right)$ and has a small apparent inner radius $\left(R_{\text {app }}<10 \mathrm{~km}\right)$, while the power law index is steep $(\Gamma \simeq 3.2)$. The shape of the energy spectrum on 1997 September 17 is characteristic of all X-ray spectra from the hard state of GRS $1915+105$ when the total flux is greater than $\simeq 4 \times 10^{-8}$ erg $\mathrm{cm}^{-2} \mathrm{~s}^{-1}$. The count rate from GRS $1915+105$ tends to vary on time scales of hours when the source is bright in X-rays, so we only integrate spectra for individual $R X T E$ orbits. With these exposure times, we can measure the power law only up to about $100 \mathrm{keV}$. However, when we combine spectra with similar photon indices, we find that the data are consistent with no cut off in the power law below $200 \mathrm{keV}$.

The changes in the X-ray spectrum clearly do not track the radio flux from GRS $1915+105$ in a monotonic manner. The power law component steepens dramatically as the radio emission increases in the $\mathrm{X}$-ray faint state (left and center panels of Figure 3), but is steepest when the Xray emission is bright and the radio emission is faint (right panel). At low X-ray fluxes $\left(<4 \times 10^{-8} \mathrm{erg} \mathrm{cm}^{-2} \mathrm{~s}^{-1}\right)$, the thermal component is hot and has a small apparent inner radius during radio plateau observations, while it is cool and has a large apparent inner radius when the radio emission is faint (see also Muno et al. 1999).

\subsubsection{Power Density Spectra and Cross Spectra}

The power spectra (here displayed as the frequency times the power density), Fourier phase lags, and coherence functions of the three observations from Figure 3 are displayed in Figure 4. In the top panel, two lines are plotted for each power spectrum: the black line indicates the $2-4.3 \mathrm{keV}$ (soft) energy band, while the the grey line indicates the 11.5-60 keV (hard) band. The PDS in all three examples of Figure 4 have relatively flat spectra with a low frequency break, a QPO between $0.5-10 \mathrm{~Hz}$, and a dramatic break at frequencies higher than the QPO. In the middle panel of Figure 4, we plot the phase lags between the $11.5-60 \mathrm{keV}$ and the $2-4.3 \mathrm{keV}$ bands, and in the bottom panel we plot the coherence function for the same two energy bands. To simplify the comparison between the power spectra, the phase lags, and the coherence functions, we have indicated the frequencies of the QPOs in our fits to the $2-4.3 \mathrm{keV}$ band with vertical black short-dashed lines, and the continuum break frequencies with vertical black long-dashed lines. We have also indicated the break frequency in the $11.5-60 \mathrm{keV}$ band with grey long-dashed lines (see below). For the following discussion we define three frequency regions related for the features of the power spectrum: region 1 represents frequencies lower than the low frequency continuum break, region 2 frequencies between the low frequency break and the QPO frequency (less its width), and region 3 frequen- 
Muno et al.

cies higher than the QPO frequency (or its harmonic if present).

The first point of interest is that the QPO frequency is not strictly correlated with the X-ray flux from GRS $1915+105$. From the spectra in Figure 3, it is clear that the X-ray flux increases from left to right, while in Figure 4 the QPO frequency is lowest in the center panel (radio plateau conditions). In the following sections, we will demonstrate that the QPO frequency is always lowest during radio plateau conditions.

The second and most obvious difference between the timing properties of these three observations is in the Fourier phase lags. In region 2 (between the low frequency break and the QPO), the phase lags are negative when the radio emission is faint (left and right panels), and positive during radio plateau emission (center panels; compare Reig et al. 2000). This behavior is in sharp contrast to sources such as GX 339-4 and Cyg X-1, for which the phase lags are always positive. Only the phase lags during the radio plateau state resemble those of GX 339-4 (Nowak et al. 1999c) and Cyg X-1 (Cui et al. 1997).

The third notable result concerns the coherence function in region 1 (i.e. frequencies lower than the first break). The coherence function between the $11.5-60 \mathrm{keV}$ and the $2-4.3 \mathrm{keV}$ bands is lowest $(\simeq 0)$ during the radio plateau conditions (center panel), somewhat higher $(\simeq 0.4)$ when both the radio and X-ray emission are faint (left panel), and nearly unity when the radio emission is faint and the $\mathrm{X}$-ray emission is bright (right panel). Only the latter case is reminiscent of Cyg X-1 in its hard state (Cui et al. 1997; Nowak et al. 1999a).

Finally, a comparison of the amplitude and strength of the power spectra as a function of energy reveals further differences in each of the three observations. While the radio emission is faint, (right and left panels) the root mean power at low frequencies is greater in the $11.5-60 \mathrm{keV}$ energy band (gray curve) than in the $2-4.3 \mathrm{keV}$ band (black curve). However, during radio plateau conditions (center panel) the root mean power is nearly equal in all energy bands. The shape of the power spectrum also changesthe low frequency break is strongest in the low energy band in the radio plateau observation (center panel), while the break is most apparent in the high energy band in the radio faint, X-ray faint observation (left panel). When the $\mathrm{X}$-ray emission is bright, the low frequency break is evident in both energy bands (right panel).

There are several other interesting details in the timing properties presented in Figure 4, although they are not directly relevant to the question of how the timing properties are related to the radio emission. The harmonic of the QPO tends to be strongest $(\simeq 10 \%)$ when count rate is lowest (left and center panels), but is not apparent during X-ray bright observations (right panel). A weak feature at approximately half of frequency of the strongest QPO peak is also evident during observations when both the $\mathrm{X}$-ray and radio flux are faint (right panel), with an RMS amplitude of about $\simeq 1 \%$ and $Q$ values of $1-10$.

Finally, a weak QPO is evident between 0.01-0.04 $\mathrm{Hz}$ during a stretch of radio faint observations from MJD 50450-40560 (left panel), with an RMS amplitude of about $0.1 \%$ in the $2-4.3 \mathrm{keV}$ energy band. This low frequency QPO disappears at higher energies. Its cen- troid frequency decreases as the frequency of the $0.5-10$ $\mathrm{Hz}$ QPO decreases (not shown). Since the 0.01-0.04 Hz $\mathrm{QPO}$ is present only in the low energy band, the coherence function at the QPO frequency is near zero.

\subsubsection{Relation of the Timing Properties to the Energy Spectrum}

The spectral fits which we have made to GRS 1915+105 indicate that two spectral components are present (Figure 3). Most models of the emission from X-ray binaries invoke inverse-Compton scattering to produce hard photons from soft seed photons, and consequently the variability in the hard and soft energy bands should be intimately related. However, before finalizing any conclusions regarding the phase lag and coherence measures and how these change with photon energy, we need to examine how these timing properties depend upon the amount of the flux which is contributed by each of the spectral components in the relevant energy bands.

In Figure 5, we examine the dependence of the timing properties on photon energy for the same three representative observations as in Figures 3 and 4 (diamonds connected by solid lines). In all of the panels, the fraction of the count rate contributed by the disk component is displayed with triangles connected with dashed lines, using the scale displayed on the right axis. Here it can be seen that the thermal component contributes no more than half of the flux in all energy bands for the observations which we analyze in this paper. Examining next the top panels, we find that the presence of the thermal component does not greatly affect the RMS power in a given energy band. In the second panels, the slopes of the phase lags show no obvious breaks; we might expect changes in the slopes if the soft and hard spectral components separately affected the phase lags. Finally, while the fraction of the flux in the thermal component increases in a similar manner during the X-ray faint, radio plateau (center panel) and the $\mathrm{X}$-ray faint, radio bright observation (left panel), the coherence function at low frequencies plummets dramatically with increasing energy in the plateau observation (center panel), but is near unity when the X-ray flux is bright (right panel). We conclude that the changes in the timing properties are not related to the fraction of the emission which can be modeled with a thermal component.

\subsection{Time Evolution of $X$-ray and Radio Properties}

The evolution of the parameters of the power spectrum, phase lags, and coherence function can be viewed as a function of time during the long periods in which GRS $1915+105$ remains in the hard-steady state. The results demonstrate that the three observations selected for Figures 3-5 are representative of the groups which we have defined. In Figure 6 we show two time intervals containing the hard-steady states of GRS $1915+105$. In the first the radio emission is faint (MJD 50400-50600, left panels), and the second coincides with strong radio plateau emission (MJD 50725-50755, right panels). In the top three panels from both time intervals we plot: a) the intensity of GRS $1915+105$ as viewed by the ASM, b) the radio flux measured with the Ryle telescope at $15.2 \mathrm{GHz}$, and c) the radio spectral index between 2.25 and $8.3 \mathrm{GHz}$ $(\alpha)$ measured with the GBI. Notice that before the period of radio-plateau emission (right panels) the radio spectral 
index increases smoothly and becomes positive (more optically thick), and then it decreases symmetrically afterwards. Optically thin radio flares that are much more prominent at 2.25 and $8.3 \mathrm{GHz}$ (not shown) occur both before and after this interval.

In panel d), we plot the frequency of the $0.5-10 \mathrm{~Hz}$ QPO. Trudolyubov et al. (1999) and Chen et al. (1997) separately established that the QPO frequency tracks the flux and the count rate in the PCA during hard-steady states similar to those displayed. However, it is clear that QPO frequency is systematically higher during radio faint emission $(>2 \mathrm{~Hz})$ than during radio plateau emission $(<2 \mathrm{~Hz})$, despite the fact that the ASM count rate is lower during the faint radio emission.

In panel e) of Figure 6 we plot the average phase lags in region 2 between the $2-4.3 \mathrm{keV}$ band and 1) the $7.8-$ $11.5 \mathrm{keV}$ band (diamonds connected by the solid line), and 2) the $11.5-60 \mathrm{keV}$ band (triangles connected by the dashed line). During radio-faint hard-steady conditions, the phase lags are consistently negative and have a value of about -0.2 radians. During radio plateau emission, the phase lags are positive and increase strongly with energy. Only during radio plateau conditions are the X-ray phase lags positive, as might be expected from a simple model which seeks to explain the timing properties using Comptonization (see Section 3).

In panel $\mathrm{f}$ ) of Figure 6 we plot the average coherence function in region 1 between the $2-4.3 \mathrm{keV}$ band and 1) the $7.8-11.5 \mathrm{keV}$ bands (diamonds connected by the solid line), and 2) the $11.5-60 \mathrm{keV}$ bands (triangles connected by the dashed line) as a function of time. The coherence over this frequency range drops dramatically when the radio emission is strong and optically thick, as shown in the representative observation of the radio plateau state in the center panel of Figure 4.

In the bottom panel we plot the total of the RMS noise at frequencies lower than the QPO (region 1 and region 2 combined). The absolute values of the RMS low frequency noise should not be compared directly from observation to observation, because as the QPO frequency varies, so does the upper limit to the frequencies which we consider. However, we have plotted the RMS low frequency noise for two energy bands: the $2-4.3 \mathrm{keV}$ band (diamonds connected by a solid line) and the $11.5-60 \mathrm{keV}$ band (triangles connected by dashed lines). It is clear that there is much more low frequency power in the $11.5-60 \mathrm{keV}$ band than in the $2-4.3 \mathrm{keV}$ band during radio-faint hard X-ray conditions, while during radio-plateau conditions, the amount of power in both energies is comparable. This can also be seen by comparing the radio plateau and X-ray-faint, radio-faint observations in Figure 4.

\subsection{Timing Properties and the Radio States}

We now examine directly the relationship between the radio and the X-ray properties of GRS $1915+105$. In Figures 7 and 8 , the various symbols denote the emission states in Figure 2.

In Figure 7 we plot as a function of the radio flux density at $15.2 \mathrm{GHz}$ : a) the QPO frequency, b) the phase lag between the $2-4.3 \mathrm{keV}$ and $11.5-60 \mathrm{keV}$ bands in region 2 , c) the coherence for the same energy bands in region 1 , and d) the ratio of the low-frequency (regions 1 and 2) power in the $11.5-60 \mathrm{keV}$ to $2-4.3 \mathrm{keV}$ energy bands. At first glance, there appear to be continuous distributions in the radio and timing properties in Figure 7 , but in fact the populations of radio faint observations and radio plateau observations are well-separated, with the radio steep observations falling in between the two. Moreover, the $x$-axes of the panels are plotted with a logarithmic scale, which emphasizes the large range in the radio flux for the various hard-steady observations.

Four conclusions can be drawn from Figure 7 regarding the changes in the timing properties as a function of the radio flux. The QPO frequency tends to decrease with increasing radio flux, and almost all of the observations with radio plateau conditions (triangles) have QPO frequencies less than $2 \mathrm{~Hz}$. Second, during radio plateau hard-steady emission the hard photons lag the soft, while during radio faint $(x$ 's) conditions the hard photons lead the soft. Third, the coherence at low frequencies is lowest during radio plateau emission. Finally, the ratio of low frequency power in $11.5-60 \mathrm{keV}$ photons to the power in $2-4.3 \mathrm{keV}$ photons is nearly unity only when the radio flux is largest, during radio plateau conditions.

Using our large dataset which incorporates a variety of radio conditions, we next examine correlations which other authors have reported to be well-defined. Two studies (Chen et al. 1997; Trudolyubov et al. 1999) have examined the frequency of the $0.5-10 \mathrm{~Hz}$ QPO as a function of the PCA count rate (MJD 50275-50333) and the 3-20 keV X-ray flux (MJD 50363-50563), respectively. Both have found significant correlations among these values. In the top panels of Figure 8 we plot the QPO frequency as a function of total X-ray flux (defined in Section 2.3; left panel), thermal flux (middle panel), and power law flux (right panel). We confirm that the frequency of the $0.5-$ $10 \mathrm{~Hz}$ QPO is tightly correlated with the X-ray flux from GRS $1915+105$, but we also find that the correlations are not single-valued. In the left panel there exists a significant population of points from radio faint observations that have QPO frequencies between $2-5 \mathrm{~Hz}$, for which the $\mathrm{X}$-ray fluxes are low compared to those expected from the general trend.

A plot of the average phase lags between 2-4.3 and 11.5$60 \mathrm{keV}$ photons in region 2 as a function of the total, thermal, and power law fluxes (bottom panels of Figure 8) reveals that there is no correlation between the X-ray flux and the sign or magnitude of the phase lags. The positive phase lags therefore appear to occur independently of any given X-ray flux level, and are better predicted by the presence of optically thick radio emission.

\subsection{Characteristics of the Faint Radio Emission}

We can use the archival VLA observations to make some conclusions about the nature of the faint radio emission. The multi-frequency radio observations of GRS $1915+105$ are listed in Table 2. The first two observations occur within a day of radio faint, X-ray bright observations. In both cases, the VLA observed significant flaring, while Ryle observations, which were more nearly simultaneous with the $R X T E$ observations, indicate that the radio emission was near $5 \mathrm{mJy}$ within 12 hours of the PCA observations. It appears that the radio emission is unsteady within several hours of the X-ray bright hard-steady state, 
and we can not rule out that the X-ray emission is also unsteady on these time scales. Since the VLA observations show clear evidence for flaring while the Ryle observations do not, we can not infer the radio spectrum of the emission during the X-ray bright, hard-steady state.

The remainder of the observations in Table 2 are X-ray faint. There is clear evidence in the GBI data for $\simeq 30$ mJy optically thin flares on 1996 December 28, 1997 January 12, and 1998 October 08. The flares decay on time scales of several hours, and no RXTE observations were taken within 0.5 day of these flares. Since GBI flux measurements below $20 \mathrm{mJy}$ are uncertain, there remain two observations during which the VLA observations demonstrate that the radio emission was faint and steady. On 1998 September 14, $15.2 \mathrm{GHz}$ Ryle and 5.0 GHz VLA observations separated by 6 hours provide a spectral index $\alpha=-0.5 \pm 0.1$, which suggests that the emission is optically thin. However, it is certainly possible that the radio flux varied by a few mJy during those several hours. Dual frequency VLA observations were made at 5.0 and 15.2 $\mathrm{GHz}$ on 1998 September 29, which indicate $\alpha=0.12 \pm 0.01$. This suggests that the emission is optically thick, similar to a weak version of the radio plateau state. However, with only a single secure measurement of $\alpha$ during the radio faint state, more observations are certainly warranted.

\section{DISCUSSION}

We begin the discussion with a summary of our three hard-steady conditions:

- Radio Faint (e.g. MJD 50450-50550; MJD 50708) The radio emission has a mean flux density of about $5 \mathrm{mJy}$ at $15.2 \mathrm{GHz}$. When the X-ray flux is faint $\left(<4 \times 10^{-8} \mathrm{erg} \mathrm{cm}^{-2} \mathrm{~s}^{-1}\right)$, the X-ray spectrum is best described as the sum of a cool $\left(T_{\text {app }}<2 \mathrm{keV}\right.$, $R_{\text {app }} \sim 60 \mathrm{~km}$ ) multi-temperature blackbody and a power law of index $\Gamma \simeq 2.1$ with a cut-off near $70 \mathrm{keV}$. When the X-ray emission is brighter (> $4 \times 10^{-8} \mathrm{erg} \mathrm{cm}^{-2} \mathrm{~s}^{-1}$ ), the X-ray spectrum is best described with a hot $(>2 \mathrm{keV})$ multi-temperature black body and a steep power law $(\Gamma \simeq 3.2)$ without a cut off below $200 \mathrm{keV}$ (see Section 2.2). The QPO frequency is greater than $2 \mathrm{~Hz}$, phase lags are negative (or, soft, indicating that hard photons precede soft photons) at intermediate continuum frequencies, the coherence at low frequencies in the continuum is $\simeq 0.5-1.0(11.5-60 \mathrm{keV}$ compared to $2-4.3 \mathrm{keV}$ ), and there is much more low frequency power in the high energy band than the low energy band.

- Radio Plateau (e.g. MJD 50730-50748) The radio emission is bright $(\sim 100 \mathrm{mJy}$ at $15 \mathrm{GHz})$ and optically thick $(\alpha>-0.2)$. The $\mathrm{X}$-ray spectrum is best described by a hot multi-temperature black body $\left(T_{\text {app }}>3 \mathrm{keV}\right.$ and $\left.R_{\text {app }}<5 \mathrm{~km}\right)$ and a power law $(\Gamma \simeq 2.5)$ which could be cut-off at $E>80 \mathrm{keV}$. The QPO frequency reaches its lowest values $(<2$ $\mathrm{Hz}$ ), phase lags are positive (hard photons lag soft photons), the coherence is low (particularly at low frequencies), and there is about equal broad band RMS power at low frequencies in all energy bands.
- Radio Steep (e.g MJD 50727-50730) These observations represent the transition into and out of radio plateau conditions. The radio flux is bright, while the radio spectrum is optically thin $(\alpha<$ $-0.2)$. The X-ray flux is often higher than during radio plateau conditions, but the spectrum is still best described by a hot multi-temperature black body $\left(T_{\text {app }}>3 \mathrm{keV}\right.$ and $R_{\text {app }}<10 \mathrm{~km}$ ) and a steep power law $(\Gamma \simeq 2.5)$ with a cut-off above $80 \mathrm{keV}$. The timing properties tend to be similar to radio faint conditions (although there are exceptions): the QPO frequency is between $2-5 \mathrm{~Hz}$, the phase lags are negative, the coherence is around 0.5 , and there is more broad band power at low frequencies in the highest energy band.

Previous observations have established the nature of the bright radio emission from GRS $1915+105$. The optically thick emission during plateau conditions has been resolved as a compact jet of relativistic electrons (Dhawan et al. 2000), similar to ones which may be present in Cyg X-1 (Stirling, Spencer, \& Garret 1998) and GX 339-4 (Corbel et al. 2000). Our observations of the faint radio emission provide some evidence that it is similar to a weak radio plateau state. Radio flares with steep spectra originate from material which has been ejected from the central source (Fender et al. 1999b; Dhawan et al. 2000), and so is most likely decoupled from the instantaneous conditions of the X-ray emitting regions. In the following discussion we will focus on comparing the radio faint and radio plateau conditions, during which the radio and $\mathrm{X}$-ray properties of GRS $1915+105$ are closely related.

The timing properties of GRS $1915+105$ - the QPO frequency, phase lags, coherence function, and broad band noise - appear to be highly dependent upon the radio conditions (Figure 7). However, it is not immediately obvious whether the changes in the timing properties are part of the mechanism which produces a compact jet, or whether they result from changes in the accretion flow induced when the compact radio jet is strong. Since the location and origin of the Comptonizing electrons which generate hard X-rays is unknown, the possible connection between these and the jet is doubly uncertain. Nevertheless, we may explore interpretations of these results using simple models for the accretion flow under consideration in the literature.

In Section 4.1, we consider the extent to which Comptonization of a soft input signal by a static corona at the base of a compact radio jet is consistent with the phase lags and X-ray spectrum of GRS $1915+105$. In Sections 4.24.3 , we examine two generic models which incorporate more elements of the accretion flow. In both models, cool $(T \sim 1 \mathrm{keV})$ thermal emission originates from an optically thick accretion disk, while the hot power law component originates from inverse Compton scattering. The first basic model places the Comptonizing electrons in a spherical corona within the inner radius of the accretion disk, while the second geometry assumes that the relativistic electrons are part of a planar corona sustained by magnetic flares above the disk.

\subsection{Generic Comptonization}


Hard phase lags are generic properties of the low-hard Xray state of black hole candidates such as Cyg X-1 (Nowak et al. 1999a) and GX 339-4 (Nowak et al. 1999c). Surprisingly, the phase lags in GRS 1915+105 change from negative to positive as the strength of the radio emission increases (Figure 7b). Since Fender et al. (1999a) have suggested that the Comptonizing corona in the hard state of black hole candidates represents the base of a compact radio jet, we feel it is important to explore the possibility that the negative phase lags during radio faint observations of GRS $1915+105$ are intrinsic to the region close to the black hole, while the hard phase lags generated during the radio plateau state are due to the Comptonization of the intrinsic input signal in a large corona at the base of the compact radio jet.

The hard phase lags in GRS $1915+105$ may constrain the structure of the Comptonizing region, because the phase is roughly constant as a function of frequency (Figure 4). This is contrary to the constant time lags that would be expected from a homogeneous corona. Instead, Kazanas, Hua, \& Titarchuk (1997) and Böttcher \& Liang (1999) have demonstrated that constant phase lags can be produced in an isotropically illuminated, spherical Compton cloud with a uniform temperature and a density which decreases with radius as $R^{-1}$, since photons have an equal probability of scattering per decade of radius in such a cloud (see Nowak et al. 1999b for further discussion of these models). High-frequency variability originates from photons which have scattered from small radii, since the signal is washed out if it scatters with long time delays. On the other hand, the time lags of low-frequency signals are dominated by photons which have scattered over large radii. This produces time lags which decrease as a function of frequency, or phase lags which are nearly constant as a function of frequency.

Several authors have pointed out that the phase lags which are observed during the hard states of black hole candidates imply large scales for inverse Compton scattering, which raises the question of how energy is transported to support a large, hot corona (Poutanen \& Fabian 1999; Nowak et al. 1999b). In the plateau state of GRS $1915+105$ a phase lag of 0.5 radians at $0.3 \mathrm{~Hz}$ (middle panel of Figure 4) would imply a light travel time of $0.3 \mathrm{~s}$, or a distance of $R \tau \simeq 8 \times 10^{9} \mathrm{~cm}$ (where $\tau$ is the optical depth of the corona). For a $10 \mathrm{M}_{\odot}$ black hole, this represents thousands of Schwarschild radii. However, this distance is much smaller than the size of the compact jet observed by Dhawan et al. (2000), $10 \mathrm{AU}=5 \times 10^{14} \mathrm{~cm}$, which is consistent with the assumption that the Comptonizing electrons reside at the base of the jet. If the Compton cloud is the base of a compact jet, this reduces the difficulty in explaining how energy is transported to support a very large corona, as the jet may carry up to $10^{39} \mathrm{erg} \mathrm{s}^{-1}$ of power to much larger radii (Falcke \& Biermann 1999).

It is possible that the radio faint states are associated with a smaller Compton corona located near the inner accretion disk. In this case, negative phase lags could be caused by any number of effects intrinsic to the accretion disk, such as waves similar to those of Nowak et al. (1999a) which propagate outward in the disk rather than inward, or a modified form the of the magnetic flares of
Poutanen \& Fabian (1999). Even if a scattering corona intercepts a significant fraction of the photons from the disk, negative phase lags could be observed so long as 1) the scattering time in the corona is much smaller than the intrinsic time lags (Miller 1995) and 2) the differences between the energies of the seed photons and the observed signals are not too large (which should be the case in GRS 1915+105, since the apparent disk temperature is $>0.8 \mathrm{keV}$; Nowak \& Vaughan 1996).

Moreover, the radius of the Compton corona can change without greatly affecting the slope and cut-off energy of the power law. The electron temperature $\left(k T_{e}\right)$ of the corona is constrained to be nearly constant by the cut-off in the power law at 70-80 keV measured during X-ray faint observations (left and center panels of Figure 3). If the temperature of the corona is fixed, a steeper power law (higher $\Gamma$ ) implies a lower optical depth (see Sunyaev \& Titarchuk 1980). Since the power law is steeper during radio plateau observations $(\Gamma \simeq 2.5)$ than during the radio faint, $\mathrm{X}$-ray faint observations $(\Gamma \simeq 2.1)$, we can suggest that the optical depth of the corona decreases and its radius increases as the compact radio jet becomes more luminous. However, the X-ray bright, radio faint observations would then have a small, optically thin corona, since the phase lags are negative and the power law is steep $(\Gamma \simeq 3.2)$ during these observations (Figures 3 and 4 ). The electron acceleration mechanism could be different in the X-ray bright hard state, as is hinted at by the absence of an observable cutoff in the power law below $200 \mathrm{keV}$.

As others have noted, estimates of the Lorentz factors for electrons emitting synchrotron emission $(\sim 400$; Falcke \& Biermann 1999) suggest energies that are much larger than that of the Comptonizing electrons. One may consider the acceleration mechanism for the jet as an undetermined structure that draws material from the Compton corona near the inner disk, as in Fender et al. (1999a). In the following two sections, we therefore briefly speculate on the implications which the simplest geometries for the Compton corona described in the literature have on the jet production mechanism.

\subsection{A Spherical Corona within the Inner Accretion Disk}

Many authors have suggested that a spherical corona is formed within the inner radius of an accretion disk, either as an advection-dominated accretion flow (ADAF; Esin, McClintock, \& Narayan 1997), or by a shock in the accretion flow (Chakrabarti \& Titarchuk 1995). The compact jet responsible for the radio emission could form from a wind emanating from an ADAF (Blandford \& Begelman 1999) or a post-shock region (Chakrabarti 1999). The distinctive feature of the spherical corona model is that the radius of the inner accretion disk is variable, which leads naturally to a scenario in which the X-ray timing and spectral properties, and the strength of the radio emission, are set by the inner radius of the accretion disk.

The X-ray timing property that is perhaps most relevant to discuss in the spherical corona model is the $0.5-10 \mathrm{~Hz}$ QPO. The QPO may represent a characteristic time scale in the accretion flow such as an acoustic (e.g. Chen \& Taam 1994; Abramowicz, Chen, \& Taam 1995), Keplerian (Rodriguez et al. 2000), or free-fall (Molteni, Sponholz, \& Chakrabarti 1996) time scale. Since the QPO peak is nar- 
row $(Q>3)$, it must originate in a localized region, as all of the time scales increase as the radius increases. Among the possibilities for the origin of such a QPO are oscillations in a shock in the accretion flow (Chakrabarti \& Manickam 2000) or a spiral density wave in the inner disk (Rodriguez et al. 2000). Since the frequency of the QPO decreases during episodes in which the inner radius of the disk is thought to increase (Belloni et al. 1997; Markwardt et al. 1999), it seems plausible that the frequency of the 0.5-10 Hz QPO in GRS $1915+105$ tracks the inner radius of the accretion disk. Figure $7 \mathrm{a}$ then implies that the radio emission is strongest when the inner disk is farthest from the source.

Nobili et al. (2000) have developed a model for the phase lags in GRS $1915+105$ by assuming a spherical corona with two temperature regions: a warm $(T \simeq 1.5 \mathrm{keV})$ outer corona and a hot $(T \simeq 15 \mathrm{keV})$ inner corona. The corona is isotropically illuminated by soft seed photons. The sign and magnitude of the phase lags are correlated with the radius of the inner accretion disk, because as the disk moves inward it compresses the corona, increasing its optical depth. Large, positive phase lags occur when the inner disk radius is large, because the hot corona has an optical depth $\tau_{\mathrm{H}} \sim 1$, while the warm corona is optically thin. When the inner radius is a factor of $\sim 3$ smaller, the optical depth of the hot corona becomes extremely high $\left(\tau_{\mathrm{H}}>100\right)$, and the soft seed photons from the disk thermalize to the temperature of the optically thick hot corona. The thermalized photons are then Compton down-scattered in the cool corona, which produces soft lags. This model does not attempt to explain the phase lags as a function of Fourier frequency, but it does predict that if the QPO frequency tracks the radius of the inner disk, negative phase lags are expected when the QPO frequency is high - exactly as Reig et al (2000) observed in GRS $1915+105$. However, a corona with an optical depth of $\tau>100$ would produce a thermal spectrum, while the energy spectrum of GRS $1915+105$ between $25-200 \mathrm{keV}$ can not be described by an extremely optically thick spectrum (e.g. a spherical Compton cloud with optical depth $\tau>5$; Sunyaev \& Titarchuk 1980) when the phase lags are negative. Further work needs to be done to reconcile the negative phase lags with the spectral shape under this type of model.

The interpretation of the coherence function and the continuum power as a function of energy is less certain. When the QPO frequency is low, the coherence at low frequencies is nearest to 0 (Figure $7 \mathrm{c}$ ) and the ratio of the low frequency continuum power at high to low energies is near 1 (Figure $7 d$ ). A coherence less than 1 can occur if the variability is the superposition of separate linear signals with different properties (Vaughan \& Nowak 1997). When the inner disk is farthest from the source (low QPO frequency) there may be more area available for multiple inputs to contribute to the variability.

The radius of the inner disk should also determine the energy spectrum under a spherical corona model. We have already discussed the model of Nobili et al. (2000). A different set of assumptions are used by, for example Esin et al. (1997) and Chakrabarti \& Titarchuk (1995), who take into account the thermal balance between the soft photons and the Comptonizing electrons. Soft spectra are formed when the inner radius of the disk is close to the last stable orbit, so that the disk produces copious amounts of seed photons which cool the corona. Hard states are formed when the inner radius of disk is far from the last stable orbit and most of the corona is photon-starved. However, if we consider the faint X-ray observations, the power law is steeper $(\Gamma \simeq 2.5)$ when the QPO frequency is lower and the disk is inferred to be farther from the black hole (radio plateau observations; Figure 3 and 4). This is opposite the sense expected, because the Compton corona should be cooled less efficiently (which implies a lower $\Gamma$ ) when the inner disk is large. If the QPO frequency tracks the transition radius between the disk and the corona, there must an additional factor beside the radius of the inner accretion disk which affects the observed X-ray properties of the corona in GRS 1915+105 under this model.

\subsection{A Planar Corona Sustained by Magnetic Flares}

In a planar corona model, one can assume that magnetic flares powered by the differential rotation of the accretion disk produce a population of relativistic electrons above the disk (di Matteo et al. 2000; compare Dove et al. 1997 for difficulties with static models). The compact jet could represent a collimated wind which forms according to the magnetic-centrifugal mechanism of Blandford \& Payne (1982), and is strongest when the flares provide electrons enough energy to escape the potential of the black hole (Romanova et al. 1998). In the magnetic flare model of di Matteo et al. (1999), the inner radius of the accretion disk in general does not vary.

A natural starting point for discussing the planar corona models are the phase lags observed in GRS 1915+105. The time lags which would be implied by the scale height of the flares in the model of di Matteo et al. (1999) are too short $\left(10^{7} \mathrm{~cm}=10^{-3} \mathrm{~s}\right)$ to explain the phase lags observed in black hole candidates. However, Poutanen \& Fabian (1999) have developed a model for Cyg X-1 in which the spectral evolution of the flares which accelerate Comptonizing electrons produce the observed power density spectra and phase lags. Their model produces only hard (or zero) lags, because the electrons are assumed to be in thermal equilibrium during the flare, so the spectrum of the flares is set by the ratio of the heating in the flares to the amount of soft seed flux impinging on the flares. The flares become harder because the majority of the soft flux is produced by feedback in response to the flare, and the feedback is taken to decrease as a function of time as the flares move away from the disk. However, if the electrons accelerated in the magnetic flare have a non-thermal energy distribution which evolves as a function of time, it is conceivable that either hard or soft phase lags could be produced. The hard phase lags may represent the most efficient acceleration of the electrons, which could in turn explain why the radio plateau emission is associated with the largest hard phase lags. On the other hand, the negative phase lags could represent spectral evolution in which the soft energy band peaks later, and relativistic electrons are accelerated less efficiently.

In the case of a magnetically sustained planar corona with a fixed inner disk radius, there is some difficulty in explaining what sets the frequency of the QPO and why this frequency varies. Since the power in the X-ray variability 
in GRS $1915+105$ drops sharply at frequencies higher than the $0.5-10 \mathrm{~Hz}$ QPO (Figure 4), we can speculate this QPO represents a resonance at the minimum time scale at which magnetic flares can form. The radio emission, then, would occur most favorably when only relatively slow flares form (low QPO frequency).

The coherence function and the ratio of the high to low energy broad-band power would also be set by the evolution of the magnetic flares. Since the coherence at low frequencies is lowest when the QPO frequency is low and the phase lags are positive, the long, hard flares may undergo significant non-linear spectral evolution. The ratio of broadband power in the high and low energy bands could be set by the shape of individual flares in the respective energy bands. Mathematically, this could be described in a manner similar to the shot noise models of Shibazaki et al. (1988).

The model of di Matteo et al. (1999) predicts the energy spectrum from magnetic flares, by assuming that a fixed fraction of the accretion power is released into the magnetic structures, and that the electrons which are accelerated by the flares are cooled inverse-Compton scattering of both cyclo-synchrotron and thermal (disk) seed photons. Soft spectral states are caused by flares which occur close to the disk and are flooded by seed photons, while hard states are caused by photon-starved flares which occur high above the disk. Although di Matteo et al. (1999) do not attempt to explain the phase lags observed in black hole candidates, we might expect hard phase lags to correspond with flares that produce the hardest (lowest $\Gamma$ ) power law, since the flares would be highest above the disk and cooled least efficiently. However, when the phase lags are positive in GRS $1915+105$ we find a steeper power law ( $\Gamma \simeq 2.5$ in the plateau state) than when the phase lags are negative $(\Gamma \simeq 2.1$ during radio faint, $\mathrm{X}$-ray faint observations). Clearly, simple assumptions about the evolution of magnetic flares would have to be relaxed in order to explain the X-ray timing and spectral properties of GRS $1915+105$.

\section{CONCLUSIONS}

We have found that although radio emission is always associated with the hard-steady state of GRS 1915+105 (between 1996-1999), the $15.2 \mathrm{GHz}$ radio flux density and the 2-200 keV X-ray flux are not correlated. Instead, we find that the hard states can be described using three basic regimes of radio emission: a) radio plateau conditions in which bright, optically thick emission originates from a compact jet, b) radio steep conditions in which bright, optically thin emission originates from material most likely decoupled from the X-ray emitting region, and c) faint radio conditions for which our current study is unable to constrain the spectrum of the radio emission. As the radio flux increases, 1) the frequency of a ubiquitous 0.5$10 \mathrm{~Hz}$ QPO decreases, 2) the Fourier phase lags between hard $(11.5-60 \mathrm{keV})$ and soft $(2-4.3 \mathrm{keV})$ photons in the frequency range of $0.01-10 \mathrm{~Hz}$ change sign from negative to positive, 3) the coherence at low frequencies decreases, and 4) the relative amount of low frequency power in hard photons compared to soft photons decreases.

We have attempted to understand these results qualitatively using simple models collected from the literature, but we find that our attempts to understand the X-ray timing properties of the hard state of GRS 1915+105 inevitably lead to contradictions with the observed energy spectrum. These observational results therefore provide impetus for developing more sophisticated models of the accretion flow and the compact jet in GRS 1915+105.

Radio astronomy at the Naval Research Laboratory is supported by the Office of Naval Research. The Green Bank Interferometer is a facility of the National Science Foundation, operated by the National Radio Astronomy Observatory in support of the NASA High Energy Astrophysics programs. The NRAO VLA is a facility of the National Science Foundation, operated under Cooperative Agreement by Associated Universities, Inc. This work was also supported in part by NASA contract NAS 5-30612. Robert Hjellming passed away in July 2000, and his contributions to astrophysics will be sorely missed.

\section{REFERENCES}

Abramowicz, M. A., Chen, X., \& Taam, R. E. 1995, ApJ, 452, 379

Bandyopadhyay, R., Martini, P., Gerard, E., Charles, P. A., Wagner, R. M., Shrader, C., Shahbaz, T., \& Mirabel, I. F. 1998, MNRAS, 295,623

Belloni, T., Klein-Wolt, M., Méndez, M., van der Klis, M., \& van Paradijs, J., 2000 A\&A, 335, 271

Belloni, T., Méndez, M., King, A. R., van der Klis, M., \& van Paradijs, J. 1997, ApJ, 488, L109

Blandford, R. D. \& Begelman, M. C. 1999, MNRAS, 303, L1

Blandford, R. D. \& Payne, D. G. 1982, MNRAS, 199, 883

Böttcher, M. \& Liang, E. P. 1998, ApJ, 506, 281

Brocksopp, C., Fender, R. P., Larionov, V., Lyuty, V. M., Tarasov, A. E., Pooley, G. G., Paciesas, W. S., \& Roche, P. 1999, MNRAS, 309, 1063

Castro-Tirado, A. J., Brandt, S., \& Lund, N. 1992, IAU Circ., 5590

Chakrabarti, S. K. 1999, A\&A, 351, 185

Chakrabarti, S. K., \& Manickam, S. G. 2000, ApJ, 531, L41

Chakrabarti, S. K. \& Titarchuk, L. G. 1995, ApJ, 455, 623

Chen, X., Swank, J. H., \& Taam, R. E. 1997, ApJ, 477, L41

Chen, X. \& Taam, R. A. 1994, ApJ, 431, 732

Corbel, S., Fender, R. P., Tzioumis, A. K., Nowak, M., McIntyre, V., Durouchoux, P., \& Sood, R. 2000, A\&A, 359, 251

Cui, W., Zhang, S. N., Focke, W., \& Swank, J. H. 1997, ApJ, 484, 383

Dhawan, V, Mirabel, I. F., \& Rodríguez, L. F. 2000, ApJ, 543, 373 di Matteo, T., Celotti, A., \& Fabian, A. C. 1999, MNRAS, 304, 809 Dove, J. B., Wilms, J., Maisack, M., \& Begelman, M. C. 1997, ApJ487, 759

Eikenberry, S. S., Matthews, K., Morgan, E. H., Remillard, R. A., \& Nelson, R. W. 1998, ApJ, 494, L61

Esin, A. A., McClintock, J. E., \& Narayan, R. 1997, ApJ, 489, 865

Falcke, H. \& Biermann, P. L., A\&A, 342, 49

Fender, R. P. 20001, MNRAS, 322, 31

Fender, R. P. et al. 1999a, ApJ, 519, L165

Fender, R. P., Garrington, S. T., McKay, D. J., Muxlow, T. W. B., Pooley, G. G., Spencer, R. E., Stirling, A. M., \& Waltman, E. B. 1999b, MNRAS, 304, 865

Foster, R. S., Waltman, E. B., Tavani, M., Harmon, B. A., Zhang, S. N., Paciesas, W. S., \& Ghigo, F. D. 1996, ApJ, 467, L81

Greiner, J., Morgan, E. H., \& Remillard, R. A. 1996, ApJ, 473, L107

Harmon, B. A., Deal, K. J., Paciesas, W. S., Zhang, S. N., Robinson, C. R., Gerard, E., Rodríguez, L. F., \& Mirabel, I. F. 1997, ApJ, $477, \mathrm{~L} 85$

Hjellming, R. M., Gibson, D. M., \& Owen, F. N. 1975, Nature, 256, 111

Jahoda, K., Swank, J. H., Giles, A. B., Stark, M. J., Strohmayer, T., Zhang, W., \& Morgan, E. H. 1996, in Proc. SPIE 2808, EUV, X-Ray, and Gamma-Ray Instrumentation for Astronomy VII, ed. O. H. Siegmund \& M. A. Gummin (Bellingham: SPIE), 59

Kazanas, D., Hua, X.-M., \& Titarchuk, L. 1997, ApJ, 480, 735 
Levine, A. M., Bradt, H., Cui, W., Jernigan, J. G., Morgan, E. H., Remillard, R., Shirey, R. E., \& Smith, D. A. 1996, ApJ, 469, L33 Magdziarz, P. \& Zdziarski, A. A. 1995, MNRAS, 273, 837

Markwardt, C. B., Swank, J. H., Taam, R. E. 1999, ApJ, 513, L37

McCollough, M. L. et al. 1999, ApJ, 517, 951

Merloni, A., Fabian, A. C., \& Ross, R. R. 2000, MNRAS, 313, 193

Miller, M. C. 1995, ApJ, 441, 770 282, L17

Mirabel, I. F., Dhawan, V., Chaty, S., Rodríguez, L. F., Marti, J., Robinson, C. R., Swank, J., Geballe, T. 1998, A\&A, 300, L9

Mirabel, I. F. \& Rodríguez, L. F. 1994, Nature, 371, 46

Mirabel, I. F. \& Rodríguez, L. F. 1999, ARA\&A, 37, 409

Molteni, D, Sponholz, H. \& Chakrabarti, S. K. 1996, ApJ, 457, 805

Morgan, E. H., Remillard, R. A., \& Greiner, J. 1997, ApJ, 482, 993

Muno, M. P., Morgan, E. H., \& Remillard, R. A. 1999, ApJ, 527, 321

Nobili, L., Turolla, R., Zampieri, L., \& Belloni, T. 2000, ApJ, 538, L137

Nowak, M. A. \& Vaughan, B. A. 1996, MNRAS, 280, 227

Nowak, M. A., Vaughan, B. A., Wilms, J., Dove, J. B., \& Begelman, M. C. 1999a, ApJ, 510, 874

Nowak, M. A., Wilms, J., Vaughan, B. A., Dove, J. B., \& Begelman, M. C. 1999b, ApJ, 515, 726

Nowak, M. A., Wilms, J., \& Dove, J. B. 1999c, ApJ, 517, 355

Orosz, J. A. \& Bailyn, C. D. 1997, ApJ, 477, 876

Orosz, J. A., et al. 2001, ApJsubmitted, astro-ph/0103045

Pooley, G. G. \& Fender, R. P. 1997 MNRAS, 292, 925

Poutanen, J. \& Fabian, A. C. 1999 MNRAS, 306, L31
Rao, A. R., Naik, S., Vadawale, S. V., \& Chakrabarti, S. K. 2000a, A\&A, 360, L25

Rao, A. R., Yadav, J. S., \& Paul, B. 2000b, ApJ, 544, 443

Reig, P., Belloni, T., van der Klis, M., Méndez, M., Kylafis, N. D., \& Ford, E. C. 2000, ApJ, 541, 883

Rodríguez, L. F, Gerard, E., Mirabel, I. F., Gómez, Y., \& Velázquez, A. 1995, ApJS, 101, 173

Rodriguez, J. Varnière, P., Tagger, M., \& Durouchoux, P. 2000, A\&A, submitted

Romanova, M. M., Ustyugova, G. V., Koldoba, A. V., Chechetkin, V. M., \& Lovelace, R. V. E. 1998, ApJ, 500, 703

Rothschild et al. 1998, ApJ, 496, 538

Shahbaz T., Groot P., Phillips S. N., Casares J., Charles P. A., \& van Paradijs J. 2000, MNRAS, 314, 747

Shibazaki, N., Elsner, R. F., Bussard, R. W., Ebisuzaki, T., \& Wiesskopf, M. C. 1988, ApJ, 331, 247

Stirling, A., Spencer, R., \& Garret, M. 1998, New Astronomy Reviews, 42, 657

Sunyaev, R. A. \& Titarchuk, L. G. 1980, A\&A, 86, 121

Tagger, M. \& Pellat, R. 1999, A\&A, 349, 1003

Taylor, G. B., Carilli, C. L., \& Perley, R. A. eds. 1999, Summer School on Synthesis Imaging, published as: ASP conf series, 1999, vol 180, ISBN 1-58381-005-6.

Trudolyubov, S., Churazov, E., \& Gilfanov, M. 1999, AstL, 25, 718

Vaughan, B. A. \& Nowak, M. A. 1997, ApJ, 474, L43

Waltman, E. B., Ghigo, F. D., Johnston, K. J., Foster, R. S., Fiedler, R. L., \& Spencer, J. H. 1995, AJ, 110, 290

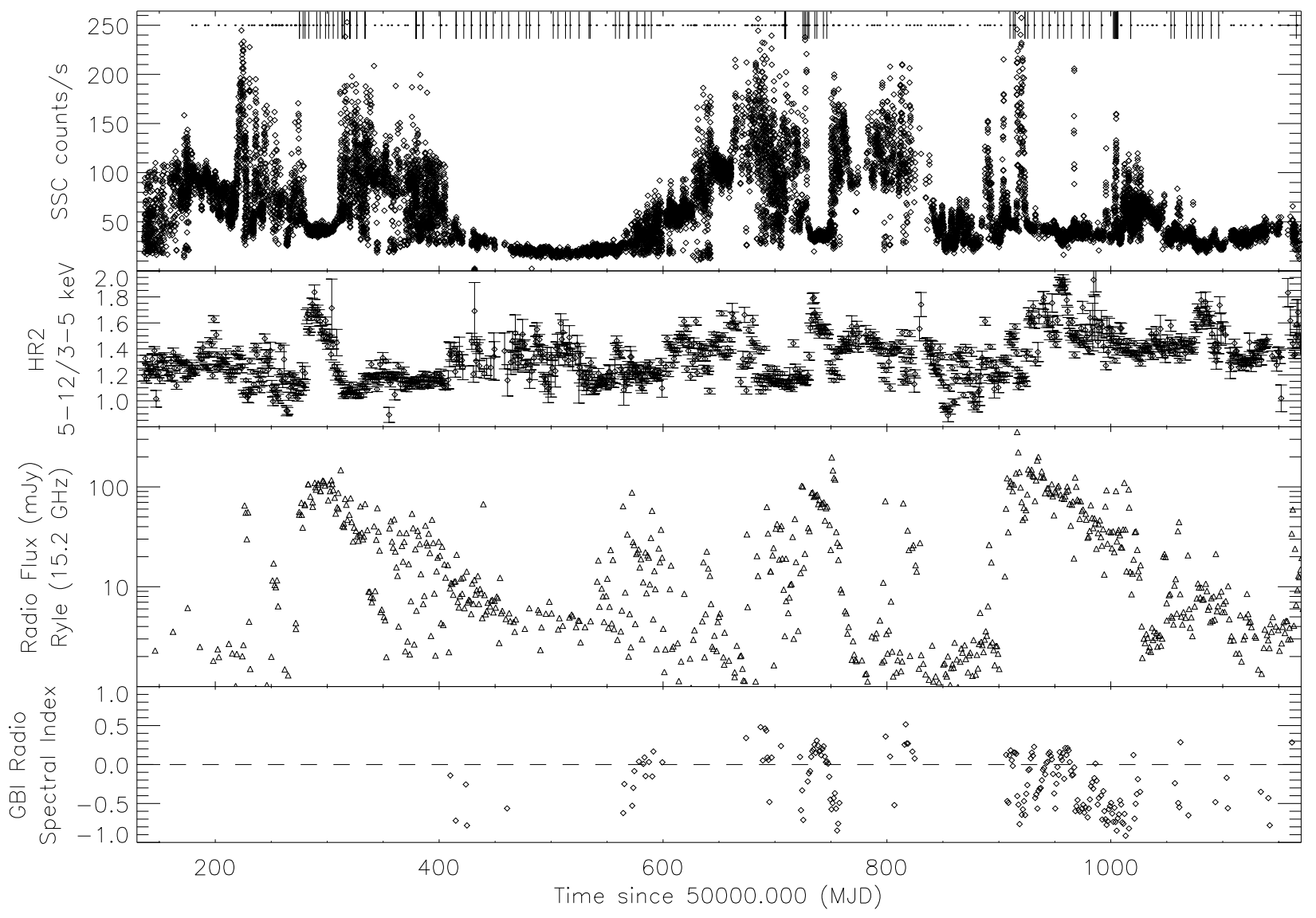

FIG. 1.- Monitoring data from X-ray and radio wavelengths. Top panel: Count rate as a function of time from the RXTE ASM, 1.5-12 $\mathrm{keV}$. Vertical bars across the top represent hard-steady pointed PCA/HEXTE observations. Dots represent the remainder of the pointed observations. Second panel: RXTE ASM hardness ratio HR2 (5-12 keV / 3-5 keV). Third panel: Flux as a function of time from the Ryle telescope at $15.2 \mathrm{GHz}$. Bottom panel: GBI radio spectral index $\left(\alpha=\Delta \log S_{\nu} / \Delta \log \nu\right.$, where $\Delta \log S_{\nu}$ is the difference between the logarithm of the mean daily flux at $\nu=8.3$ and $2.25 \mathrm{GHz}$; points are plotted only if the flux density at both frequencies was larger than 20 mJy). 


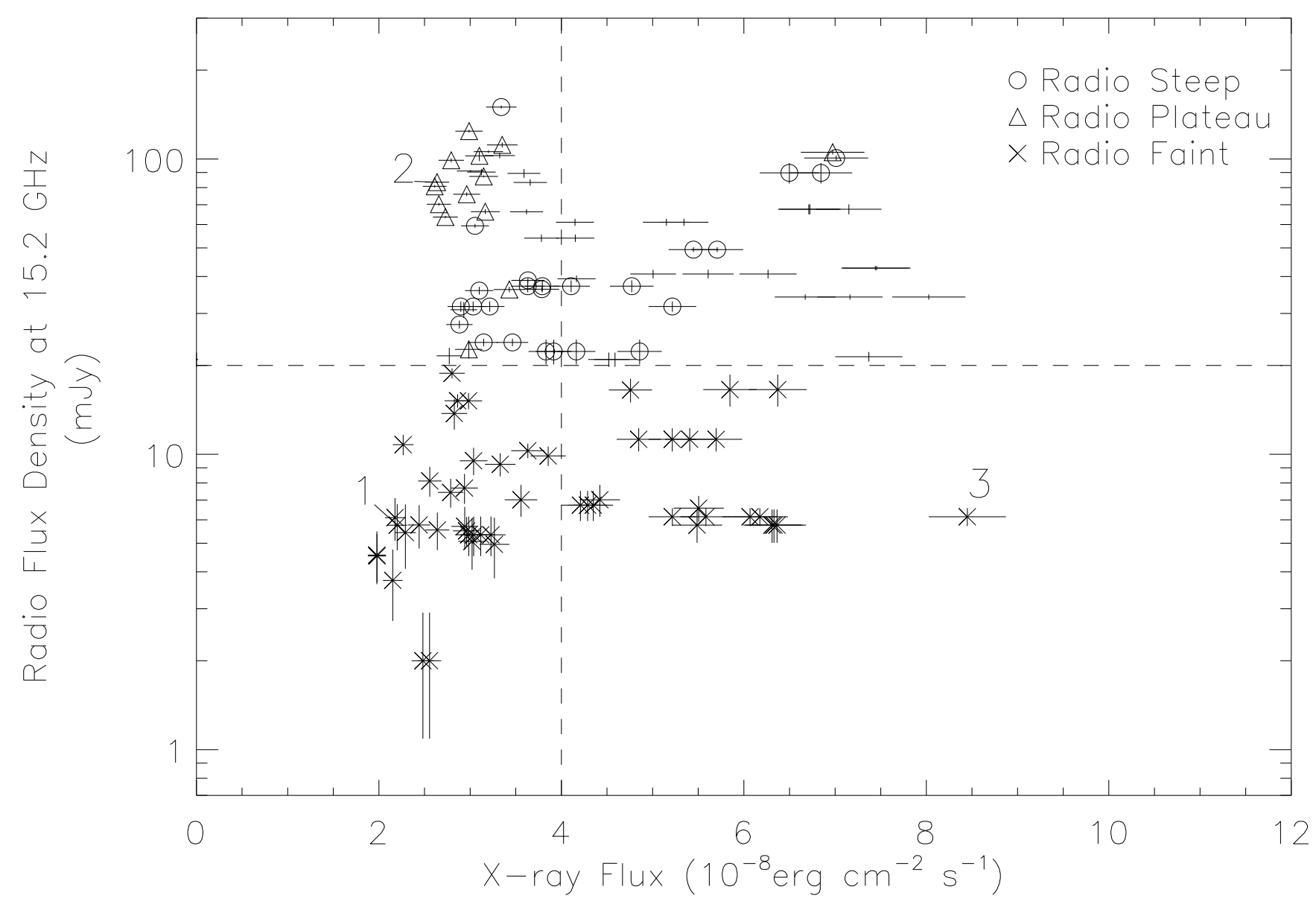

FIG. 2.- The radio flux density at $15.2 \mathrm{GHz}$ measured with the Ryle telescope as a function of the 2-200 keV X-ray flux measured with the PCA aboard RXTE. All hard-steady observations exhibit detectable radio emission, although the strength of the X-ray and radio emission are not correlated. The radio conditions of each observation are indicated as follows: triangles represent radio-plateau hard-steady conditions, circles radio-steep conditions, and $X$ 's radio-faint conditions. Three characteristic observations are indicated in the diagram: (1) a radio-faint, X-ray faint observation, (2) a radio plateau, X-ray faint observation, and (3) a radio-faint, X-ray bright observation. 


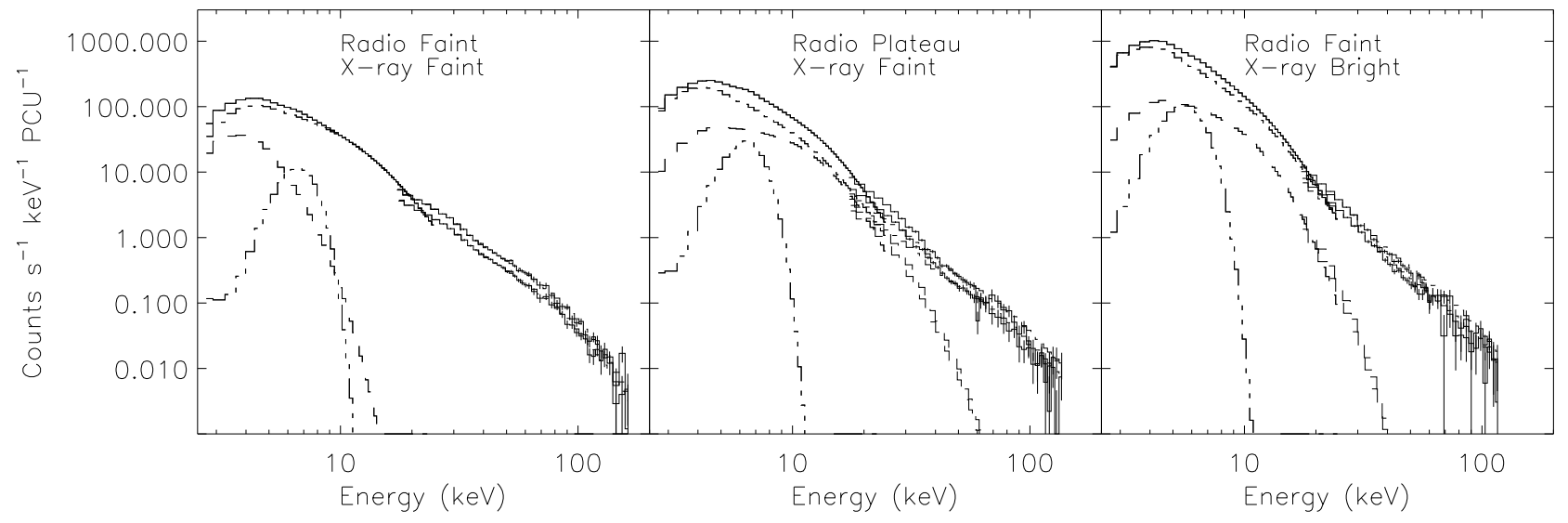

FIG. 3. - The energy spectrum and the corresponding spectral fits, as described in the text, for three characteristic observations: a radiofaint and X-ray faint observation on MJD 50488, a radio-plateau observation on MJD 50737, and a radio-faint and X-ray bright observation on MJD 50708. The power law component dominates the spectrum at all energies, while the multi-temperature disk and the Gaussian iron line contribute a smaller fraction of the flux. 

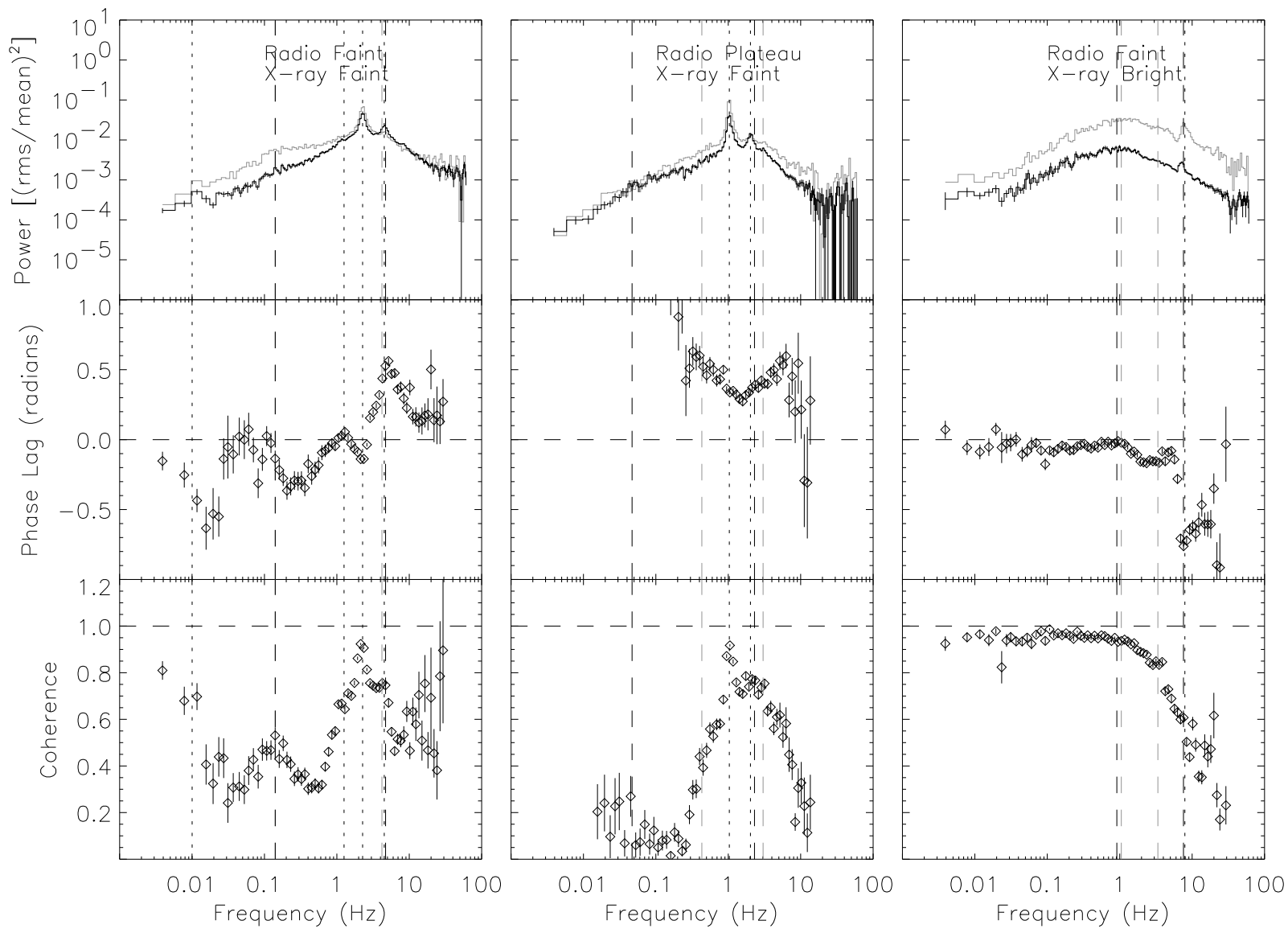

FIG. 4.- Timing properties of the three characteristic observations in Figure 3. Top panel: PDS in two energy bands, the black line indicates the $2-4.3 \mathrm{keV}$ energy band, while the grey line indicates the $11.5-60 \mathrm{keV}$ band. Middle panel: the phase lags between the $2-4.3 \mathrm{keV}$ and the $11.5-60 \mathrm{keV}$ bands. Bottom panel: the coherence function between the $2-4.3 \mathrm{keV}$ and the $11.5-60 \mathrm{keV}$ bands. In all panels, we have indicated the frequencies of the QPOs in our fits to the $2.5-5 \mathrm{keV}$ band with black short-dashed lines, and the break frequencies with black long-dashed lines. We have also indicated the break frequency in the 11.5-60 keV band with grey long-dashed lines (see below). 


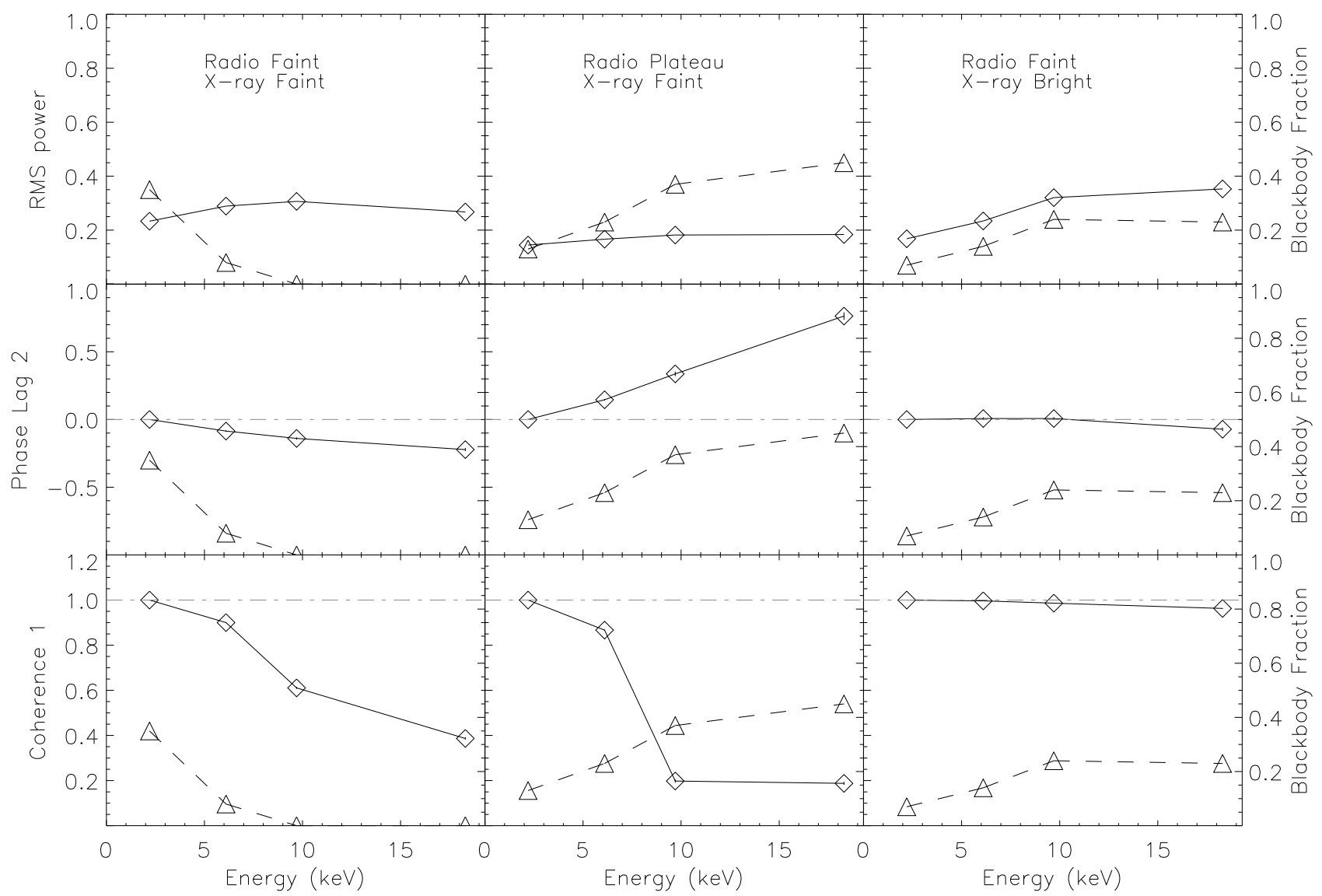

FIG. 5.- The relation of the timing properties to the X-ray energy spectrum for the three observations in Figure 3. Top panels: The RMS variability at all frequencies as a function of energy plotted with diamonds connected by the solid line. Middle panels: The mean phase lag between the low frequency break and the QPO frequency (region 2) as a function of energy plotted with diamonds connected by the solid line. Positive values indicate that the hard photons lag the soft photons. Bottom panels: The average coherence function measured below the low frequency break (region 1) plotted with diamonds connected by the solid line. All Panels: Fraction of the count rate contributed by the multi-temperature disk model (as derived from the spectral fits) as a function of energy, plotted as triangles connected by the dashed line, and labeled on the axes to the right. 


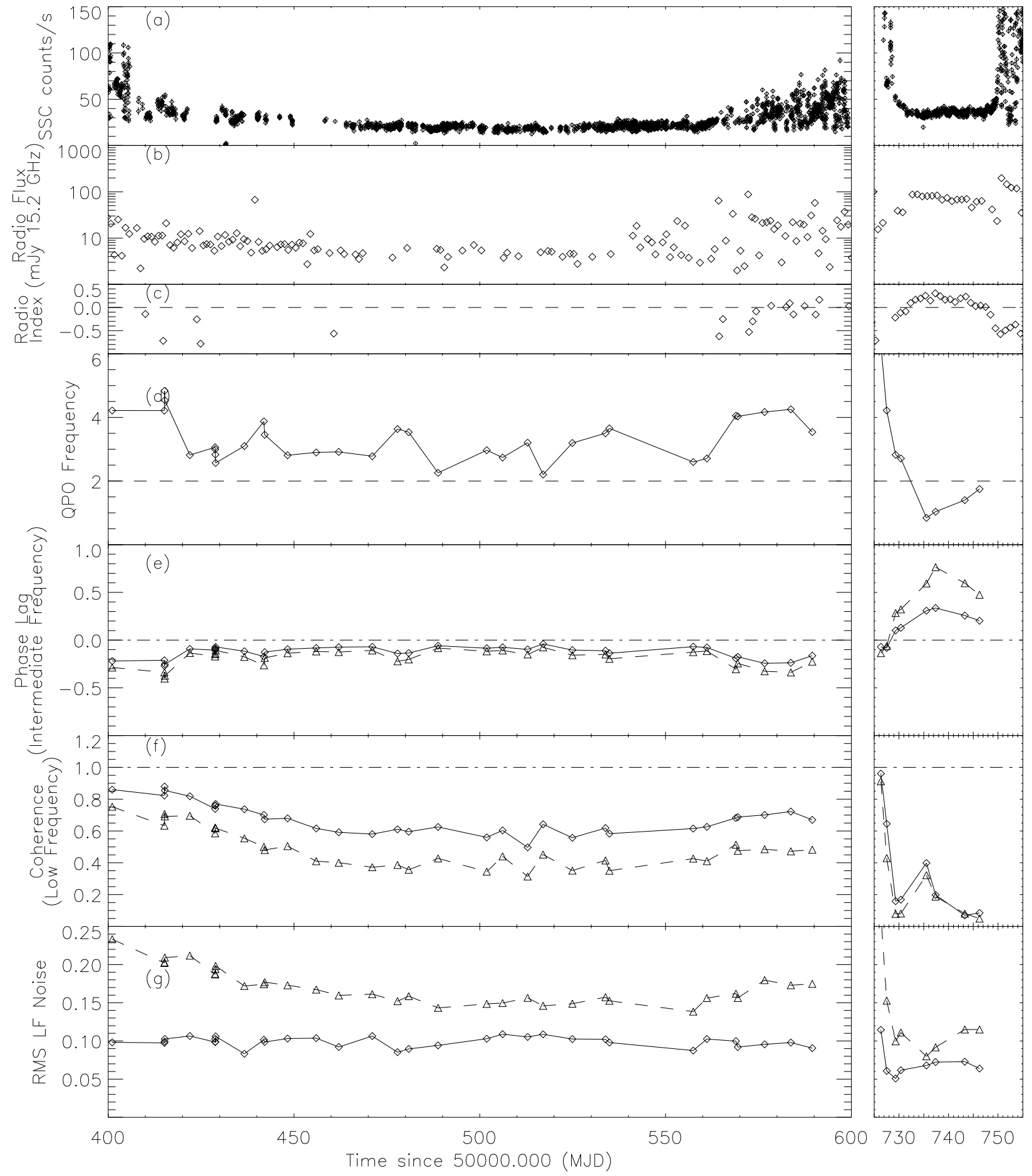

FIG. 6.- The time evolution of the parameters of the PDS and CS for a stretch of faint radio emission (MJD 50400-50600, left panel) and bright radio emission (MJD 50725-50755, right panel). a) The ASM light curve, 1.5-12 keV. b) Ryle monitoring data (15.2 GHz). c) The radio spectral index $(\alpha)$ measured by with the GBI (see Figure 1). d) The $0.5-10 \mathrm{~Hz}$ QPO frequency (measured from the $2-4.3$ keV band). e) Phase lags for the $2-4.3 \mathrm{keV}$ relative to the $7.8-11.5 \mathrm{keV}$ band (diamonds connected with the solid line) and the 11.5-60 keV band (triangles connected with the dashed line) measured between the low frequency break and the QPO. f) The average coherence function for the $2-4.3 \mathrm{keV}$ relative to the $7.8-11.5 \mathrm{keV}$ band (diamonds connected with the solid line) and the $11.5-60 \mathrm{keV}$ band (triangles connected with the dashed line) measured below the low frequency break. g) The RMS power at frequencies below the QPO measured in the 2-4.3 keV band (diamonds connected with the solid line) and in the 11.5-60 keV (triangles connected with the dashed line). 

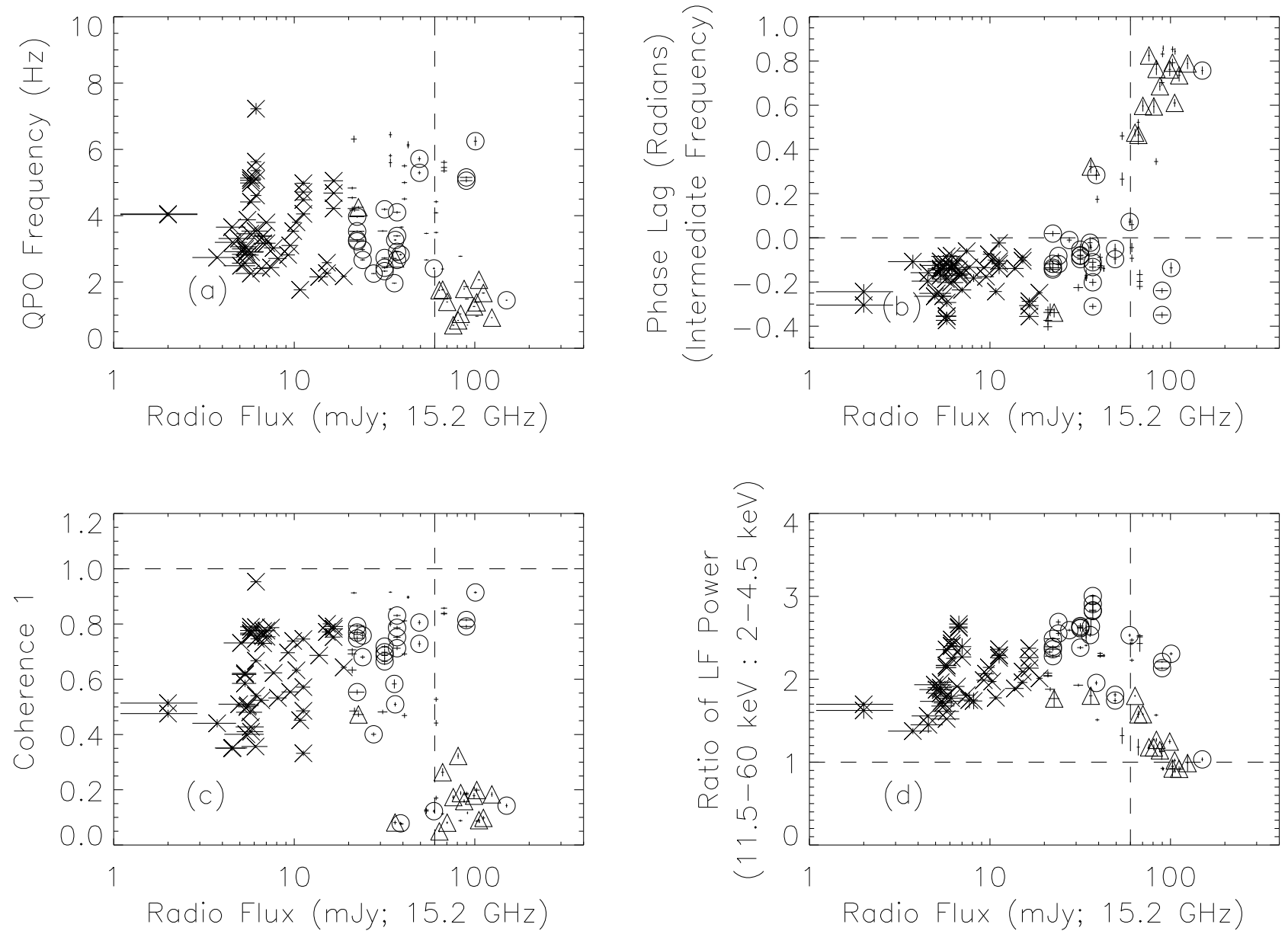

FIG. 7.- Parameters of the PDS and CS as a function of the radio flux (15.2 GHz). Triangles represent radio-plateau hard-steady conditions, circles radio-steep conditions, and X's radio-faint conditions. a) The QPO frequency. b) Phase lags for 11.5-60 keV photons relative to $2-4.3 \mathrm{keV}$ photons, averaged over intermediate frequencies (between the low frequency break and the QPO). c) The coherence function for $2-4.3 \mathrm{keV}$ photons relative to $11.5-60 \mathrm{keV}$ photons, averaged over low frequencies (below the low frequency break). d) The ratio of the integrated broad band powers below the QPO frequency in the high (11.5-60 keV) to low (2-4.3 keV) energy bands. 

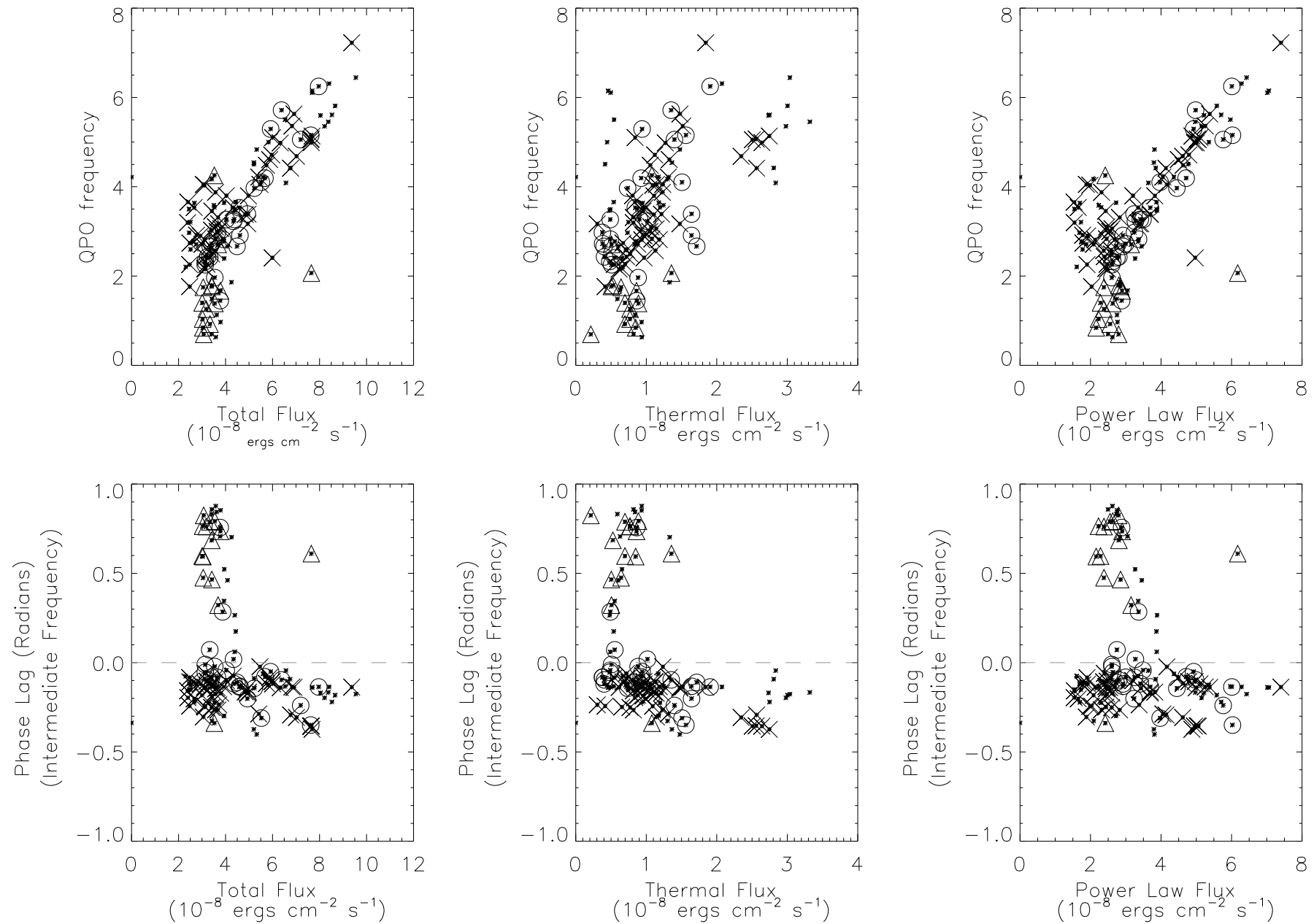

FIG. 8.- Correlations between the QPO frequency (top panels) or the average phase lag at intermediate frequencies (11.5-60 keV relative to $2-4.3 \mathrm{keV}$; bottom panels) with fluxes derived from spectral fits to the data. Left panels: total flux. Center panels: thermal flux, from the multi-temperature disk model. Right panels: power law flux, integrated from 2-200 keV. The symbols are the same as Figure 7 . 
TABLE 1

Hard-Steady Pointed RXTE Observations of GRS $1915+105$

\begin{tabular}{|c|c|c|c|c|c|c|}
\hline ObsID & $\begin{array}{l}\text { Date } \\
\text { (UT) }\end{array}$ & $\begin{array}{l}\text { X-ray } \\
\text { Flux }^{a}\end{array}$ & $\mathrm{HR}^{\mathrm{b}}$ & $\begin{array}{c}S_{2.25} \\
(\mathrm{mJy})\end{array}$ & $\begin{array}{c}S_{8.3} \\
(\mathrm{mJy})\end{array}$ & $\begin{array}{c}S_{15.2} \\
(\mathrm{mJy})\end{array}$ \\
\hline 10408-01-22-00 & 1996 Jul 11 02:09 & $4.15(1)$ & 0.063 & $\cdots$ & $\cdots$ & $54(2)$ \\
\hline $10408-01-22-01$ & 1996 Jul 11 05:16 & $3.93(1)$ & 0.070 & $\cdots$ & $\cdots$ & $54(2)$ \\
\hline $10408-01-22-02$ & 1996 Jul 11 08:38 & $3.78(1)$ & 0.073 & $\cdots$ & $\ldots$ & $54(2)$ \\
\hline $10408-01-23-00$ & 1996 Jul 14 11:45 & $4.17(1)$ & 0.063 & $\cdots$ & $\cdots$ & $39.3(6)$ \\
\hline $10408-01-24-00$ & 1996 Jul 16 04:04 & $3.62(1)$ & 0.075 & $\cdots$ & $\cdots$ & $66(1)$ \\
\hline $10408-01-25-00$ & 1996 Jul 19 11:45 & $3.20(1)$ & 0.101 & $\cdots$ & $\cdots$ & $105.9(7)$ \\
\hline $10258-01-01-00^{\mathrm{c}}$ & 1996 Jul 23 10:33 & $3.54(1)$ & 0.118 & $\ldots$ & $\ldots$ & $65.8(7)$ \\
\hline $10408-01-27-00$ & 1996 Jul 26 13:55 & $3.12(1)$ & 0.114 & $\ldots$ & $\ldots$ & $\ldots$ \\
\hline $10258-01-02-00$ & 1996 Jul 29 18:28 & $3.00(1)$ & 0.111 & $\cdots$ & $\ldots$ & $91.1(6)$ \\
\hline $10408-01-28-00$ & 1996 Aug 03 12:43 & $3.32(1)$ & 0.105 & $\cdots$ & $\cdots$ & $103(2)$ \\
\hline $10258-01-03-00$ & 1996 Aug 06 07:12 & $3.12(1)$ & 0.096 & $\cdots$ & $\cdots$ & $90.3(4)$ \\
\hline $10408-01-29-00$ & 1996 Aug 10 08:52 & $3.59(1)$ & 0.090 & $\cdots$ & $\cdots$ & $89.4(6)$ \\
\hline 10258-01-04-00 & 1996 Aug 14 12:14 & $3.66(1)$ & 0.075 & $\cdots$ & $\cdots$ & $83.3(5)$ \\
\hline $10408-01-30-00$ & 1996 Aug 18 07:26 & $5.01(1)$ & 0.058 & $\cdots$ & $\cdots$ & $40.8(6)$ \\
\hline 10258-01-05-00 & 1996 Aug 20 07:40 & $7.46(1)$ & 0.055 & $\cdots$ & $\ldots$ & $42.7(5)$ \\
\hline $10408-01-31-00$ & 1996 Aug 25 04:47 & $5.15(1)$ & 0.065 & $\ldots$ & $\ldots$ & $61.1(5)$ \\
\hline 10258-01-06-00 & 1996 Aug 29 11:31 & $8.66(1)$ & 0.058 & $\cdots$ & $\cdots$ & $35.5(8)$ \\
\hline $10408-01-32-00$ & 1996 Aug 31 07:55 & $8.03(1)$ & 0.058 & $\cdots$ & $\cdots$ & $34.1(4)$ \\
\hline 10408-01-33-00 & 1996 Sep 07 18:00 & $6.71(1)$ & 0.063 & $\ldots$ & $\ldots$ & $67.6(5)$ \\
\hline $10408-01-42-00$ & 1996 Oct 23 03:36 & $6.37(1)$ & 0.079 & $\ldots$ & $\ldots$ & $16.6(4)$ \\
\hline $10408-01-43-00$ & 1996 Oct 23 12:00 & $5.85(1)$ & 0.079 & $\cdots$ & $\cdots$ & $16.6(4)$ \\
\hline $10408-01-45-00$ & 1996 Oct 29 12:00 & $4.21(1)$ & 0.096 & $\cdots$ & $\cdots$ & $6.7(6)$ \\
\hline 20402-01-01-00 & 1996 Nov 07 05:45 & $5.98(1)$ & 0.078 & $\cdots$ & $\cdots$ & $31.8(8)$ \\
\hline 20402-01-02-01 & 1996 Nov 14 02:09 & $4.76(1)$ & 0.090 & $\ldots$ & $\ldots$ & $16.5(5)$ \\
\hline 20402-01-02-02 & 1996 Nov 14 15:07 & $5.76(1)$ & 0.077 & $\ldots$ & $\ldots$ & $4.3(9)$ \\
\hline 20402-01-04-00 & 1996 Nov 28 03:21 & $4.95(1)$ & 0.086 & $\cdots$ & $\cdots$ & $21(1)$ \\
\hline 20402-01-05-00 & 1996 Dec 04 23:31 & $3.33(1)$ & 0.116 & $\ldots$ & $\ldots$ & $9.2(6)$ \\
\hline 20402-01-06-00 & 1996 Dec 11 18:43 & $3.23(1)$ & 0.119 & $10(4)$ & $7(6)$ & $5.3(7)$ \\
\hline 20402-01-07-00 & 1996 Dec 19 15:50 & $3.04(1)$ & 0.115 & $24(4)$ & $11(6)$ & $9.5(6)$ \\
\hline 20402-01-08-00 & 1996 Dec 24 22:04 & $2.96(1)$ & 0.102 & $16(4)$ & $10(6)$ & $5.5(5)$ \\
\hline 20402-01-08-01 & 1996 Dec 25 02:52 & $2.94(1)$ & 0.113 & $15(4)$ & $8(6)$ & $5.7(7)$ \\
\hline 20402-01-09-00 & 1996 Dec 31 06:43 & $2.64(1)$ & 0.124 & $16(4)$ & $5(6)$ & $5.5(7)$ \\
\hline $20402-01-10-00$ & 1997 Jan 07 23:45 & $2.44(1)$ & 0.125 & $5(4)$ & $5(6)$ & $5.8(8)$ \\
\hline 20402-01-11-00 & 1997 Jan 14 01:26 & $2.29(1)$ & 0.124 & $12(4)$ & $7(6)$ & $5(1)$ \\
\hline $20402-01-12-00$ & 1997 Jan 23 01:40 & $2.20(1)$ & 0.125 & $9(4)$ & $7(6)$ & $\ldots$ \\
\hline $20402-01-13-00$ & 1997 Jan 29 20:52 & $2.20(1)$ & 0.110 & $7(4)$ & $7(6)$ & $\ldots$ \\
\hline $20402-01-14-00$ & 1997 Feb 01 21:07 & $2.18(1)$ & 0.111 & $10(4)$ & $5(6)$ & $6.1(8)$ \\
\hline 20402-01-15-00 & 1997 Feb 09 18:43 & $2.20(1)$ & 0.144 & $\cdots$ & $\cdots$ & $5.8(7)$ \\
\hline 20402-01-16-00 & 1997 Feb 22 21:07 & $2.08(1)$ & 0.131 & $9(4)$ & $7(6)$ & $\ldots$ \\
\hline 20402-01-17-00 & 1997 Feb 27 03:36 & $2.15(1)$ & 0.131 & $13(4)$ & $7(6)$ & $3.7(8)$ \\
\hline $20402-01-18-00$ & 1997 Mar 05 21:21 & $2.13(1)$ & 0.120 & $10(4)$ & $8(6)$ & $\ldots$ \\
\hline 20402-01-19-00 & 1997 Mar 10 01:12 & $1.98(1)$ & 0.146 & $13(4)$ & $6(6)$ & $\ldots$ \\
\hline 20402-01-20-00 & 1997 Mar 17 22:04 & $1.98(1)$ & 0.121 & $9(4)$ & $5(6)$ & $4.6(8)$ \\
\hline 20402-01-21-00 & 1997 Mar 26 20:09 & $2.01(1)$ & 0.114 & $10(4)$ & $9(6)$ & $\ldots$ \\
\hline 20402-01-21-01 & 1997 Mar 27 21:36 & $1.98(1)$ & 0.110 & $16(4)$ & $6(6)$ & $4.5(7)$ \\
\hline 20402-01-25-00 & 1997 Apr 19 10:47 & $2.16(1)$ & 0.133 & $6(4)$ & $7(6)$ & $\cdots$ \\
\hline 20402-01-24-00 & 1997 Apr 23 03:07 & $2.33(1)$ & 0.132 & $9(4)$ & $7(6)$ & $\ldots$ \\
\hline 20402-01-26-01 & 1997 Apr 30 22:33 & $2.48(1)$ & 0.100 & $8(4)$ & $10(6)$ & $2.0(7)$ \\
\hline 20402-01-26-02 & 1997 May 01 10:47 & $2.55(1)$ & 0.103 & $8(4)$ & $9(6)$ & $2.0(7)$ \\
\hline $20402-01-27-01$ & 1997 May 08 16:04 & $2.77(1)$ & 0.097 & $15(4)$ & $14(6)$ & $22(1)$ \\
\hline $20402-01-27-03^{\mathrm{c}}$ & 1997 May 15 05:16 & $2.43(1)$ & 0.113 & $22(4)$ & $46(6)$ & $\ldots$ \\
\hline $20187-02-02-00$ & 1997 May 15 11:31 & $2.98(1)$ & 0.097 & $27(4)$ & $30(6)$ & $22.7(8)$ \\
\hline 20402-01-29-00 & 1997 May 21 11:45 & $2.93(1)$ & 0.107 & $13(4)$ & $11(6)$ & $30.9(8)$ \\
\hline 20186-03-02-05 & 1997 Sep 17 14:09 & $8.45(1)$ & 0.057 & $11(4)$ & $11(6)$ & $6.1(3)$ \\
\hline 20186-03-02-06 & 1997 Sep 18 03:07 & $5.70(1)$ & 0.062 & $10(4)$ & $10(6)$ & $11.2(3)$ \\
\hline $20402-01-47-01^{\mathrm{c}}$ & 1997 Sep 19 00:00 & $6.14(1)$ & 0.055 & $15(4)$ & $14(6)$ & $19.3(6)$ \\
\hline $20187-02-03-00$ & 1997 Oct 03 20:38 & $7.82(1)$ & 0.063 & $280(7)$ & $108(7)$ & 101(1) \\
\hline
\end{tabular}


TABLE $1-$ Continued

\begin{tabular}{|c|c|c|c|c|c|c|}
\hline ObsID & $\begin{array}{l}\text { Date } \\
\text { (UT) }\end{array}$ & $\begin{array}{c}\text { X-ray } \\
\text { Flux }^{a}\end{array}$ & $\mathrm{HR}^{\mathrm{b}}$ & $\begin{array}{c}S_{2.25} \\
\text { (mJy) }\end{array}$ & $\begin{array}{c}S_{8.3} \\
(\mathrm{mJy})\end{array}$ & $\begin{array}{c}S_{15.2} \\
(\mathrm{mJy})\end{array}$ \\
\hline 20187-02-04-00 & 1997 Oct 05 08:23 & $7.37(1)$ & 0.057 & $32(4)$ & $13(6)$ & $21.4(6)$ \\
\hline 20187-02-05-00 & 1997 Oct 06 11:45 & $5.01(1)$ & 0.062 & $32(4)$ & $16(6)$ & $\ldots$ \\
\hline 20187-02-06-00 & 1997 Oct 07 10:05 & $5.84(1)$ & 0.060 & $25(4)$ & $12(6)$ & $\ldots$ \\
\hline 20402-01-49-00 & 1997 Oct 08 07:55 & $3.63(1)$ & 0.080 & $33(4)$ & $22(6)$ & $38.8(5)$ \\
\hline 20402-01-49-01 & 1997 Oct 09 09:21 & $3.43(1)$ & 0.082 & $44(4)$ & $35(6)$ & $36.2(5)$ \\
\hline 20402-01-50-00 & 1997 Oct 14 13:12 & $2.61(1)$ & 0.124 & $50(4)$ & $65(6)$ & $80.8(5)$ \\
\hline 20402-01-50-01 & 1997 Oct 16 09:36 & $2.64(1)$ & 0.117 & $50(4)$ & $74(6)$ & $83.5(6)$ \\
\hline $20402-01-51-00$ & 1997 Oct $2206: 57$ & $2.66(1)$ & 0.113 & $45(4)$ & $65(6)$ & $70.3(7)$ \\
\hline 20402-01-52-00 & 1997 Oct $2506: 28$ & $2.73(1)$ & 0.108 & $46(4)$ & $41(6)$ & $63.6(5)$ \\
\hline $30703-01-14-00$ & 1998 Apr 06 21:07 & $3.35(1)$ & 0.092 & $78(4)$ & $91(6)$ & $111.6(9)$ \\
\hline 30402-01-09-00 & 1998 Apr 09 21:07 & $3.54(1)$ & 0.087 & $87(4)$ & $85(6)$ & $105(1)$ \\
\hline 30402-01-09-01 & 1998 Apr 10 00:00 & $3.58(1)$ & 0.086 & $87(4)$ & $85(6)$ & $105(1)$ \\
\hline 30402-01-10-00 & 1998 Apr 11 09:21 & $3.46(1)$ & 0.089 & $100(4)$ & $122(7)$ & $\ldots$ \\
\hline $30402-01-11-00$ & 1998 Apr 20 06:14 & $5.45(1)$ & 0.052 & $188(5)$ & $80(6)$ & $49.3(6)$ \\
\hline 30703-01-15-00 & 1998 Apr 22 21:21 & $3.34(1)$ & 0.094 & $132(4)$ & $100(6)$ & $150(1)$ \\
\hline $30703-01-16-00$ & 1998 Apr 28 16:19 & $3.10(1)$ & 0.101 & $82(4)$ & $111(7)$ & $102.5(6)$ \\
\hline 30703-01-17-00 & 1998 May 05 21:36 & $2.99(1)$ & 0.112 & $145(5)$ & $116(7)$ & 124(1) \\
\hline 30402-01-12-00 & 1998 May 11 22:33 & $3.18(1)$ & 0.093 & $70(4)$ & $87(6)$ & $87.3(4)$ \\
\hline 30402-01-12-01 & 1998 May 12 00:28 & $3.15(1)$ & 0.098 & $70(4)$ & $87(6)$ & $87.3(4)$ \\
\hline $30402-01-12-02$ & 1998 May 12 02:09 & $3.17(1)$ & 0.099 & $70(4)$ & $87(6)$ & $87.3(4)$ \\
\hline $30402-01-12-03$ & 1998 May 12 03:50 & $3.17(1)$ & 0.101 & $70(4)$ & $87(6)$ & $87.3(4)$ \\
\hline 30703-01-19-00 & 1998 May 19 14:23 & $2.79(1)$ & 0.112 & $99(4)$ & $95(6)$ & $99(1)$ \\
\hline $30703-01-20-00$ & 1998 May 24 19:26 & $2.96(1)$ & 0.135 & $49(4)$ & $53(6)$ & $76.0(9)$ \\
\hline $30703-01-21-00$ & 1998 May 31 19:26 & $3.17(1)$ & 0.098 & $52(4)$ & $46(6)$ & $66.3(8)$ \\
\hline 30402-01-14-00 & 1998 Jun 11 08:09 & $3.05(1)$ & 0.096 & $104(4)$ & $53(6)$ & $59.4(6)$ \\
\hline 30703-01-18-00 & 1998 Jun 16 18:14 & $3.79(1)$ & 0.087 & $91(4)$ & $43(6)$ & $36.2(5)$ \\
\hline $30703-01-22-00$ & 1998 Jun 27 14:38 & $2.93(1)$ & 0.105 & $46(4)$ & $27(6)$ & $27.5(5)$ \\
\hline $30703-01-22-01$ & 1998 Jun 27 16:33 & $2.94(1)$ & 0.106 & $46(4)$ & $27(6)$ & $27.5(5)$ \\
\hline 30182-01-01-01 & 1998 Jul 08 04:04 & $2.79(1)$ & 0.125 & $87(4)$ & $33(6)$ & $35.8(9)$ \\
\hline 30182-01-01-00 & 1998 Jul 08 05:02 & $2.80(1)$ & 0.116 & $87(4)$ & $33(6)$ & $35.8(9)$ \\
\hline $30182-01-02-01^{\mathrm{c}}$ & 1998 Jul 9 04:04 & $3.92(1)$ & 0.109 & $200(6)$ & $97(6)$ & $22.3(9)$ \\
\hline 30182-01-02-00 & 1998 Jul 09 05:02 & $4.16(1)$ & 0.098 & $200(6)$ & $97(6)$ & $22.3(9)$ \\
\hline $30182-01-03-01^{\mathrm{c}}$ & 1998 Jul 10 04:05 & $7.84(1)$ & 0.074 & $430(7)$ & $137(9)$ & $152(1)$ \\
\hline 30182-01-03-00 & 1998 Jul 10 10:05 & $7.10(1)$ & 0.079 & $350(7)$ & 139(9) & $90(1)$ \\
\hline 30182-01-04-00 & 1998 Jul 11 05:45 & $4.77(1)$ & 0.090 & $143(5)$ & $47(6)$ & $37.1(4)$ \\
\hline 30182-01-04-02 & 1998 Jul 12 04:04 & $3.46(1)$ & 0.102 & $93(4)$ & $38(6)$ & $23.9(5)$ \\
\hline 30182-01-04-01 & 1998 Jul 12 05:02 & $3.15(1)$ & 0.103 & $93(4)$ & $38(6)$ & $23.9(5)$ \\
\hline 30703-01-25-00 & 1998 Jul 23 18:00 & $3.56(1)$ & 0.103 & $28(4)$ & $12(6)$ & $7.0(7)$ \\
\hline $30402-01-16-00$ & 1998 Aug 28 16:47 & $3.02(1)$ & 0.118 & $16(4)$ & $11(6)$ & $5.1(9)$ \\
\hline 30703-01-31-00 & 1998 Aug 31 18:28 & $3.63(1)$ & 0.106 & $40(4)$ & $28(6)$ & $10.3(5)$ \\
\hline 30402-01-17-00 & 1998 Sep 11 15:07 & $3.35(1)$ & 0.112 & $17(4)$ & $10(6)$ & $\ldots$ \\
\hline 30703-01-33-00 & 1998 Sep 15 22:47 & $3.27(1)$ & 0.113 & & $\ldots$ & $5(1)$ \\
\hline 30703-01-34-00 & 1998 Sep 22 00:14 & $2.27(1)$ & 0.146 & $13(4)$ & $15(6)$ & $10.8(7)$ \\
\hline 30703-01-35-00 & 1998 Sep 25 19:40 & $2.79(1)$ & 0.136 & $12(4)$ & $9(6)$ & $7.4(7)$ \\
\hline 30703-01-36-00 & 1998 Oct 03 17:02 & $2.94(1)$ & 0.125 & $28(4)$ & $10(6)$ & $7.7(7)$ \\
\hline 30402-01-18-00 & 1998 Oct 10 08:23 & $2.56(1)$ & 0.114 & $15(4)$ & $9(6)$ & $8.1(8)$ \\
\hline $30703-01-24-01$ & 1998 Dec 18 16:47 & $2.92(1)$ & 0.129 & $12(4)$ & $13(6)$ & $6.6(6)$ \\
\hline $30703-01-24-02$ & 1998 Dec 18 19:26 & $2.77(1)$ & 0.129 & $12(4)$ & $13(6)$ & $6.6(6)$ \\
\hline 30703-01-24-00 & 1998 Dec 19 03:21 & $2.81(1)$ & 0.135 & $12(4)$ & $13(6)$ & $6.6(6)$ \\
\hline 30703-01-41-00 & 1998 Dec 26 16:04 & $2.83(1)$ & 0.139 & $16(4)$ & $9(6)$ & $14(1)$ \\
\hline 40703-01-01-00 & 1999 Jan 01 01:26 & $2.86(1)$ & 0.136 & $37(4)$ & $16(6)$ & $15.2(7)$ \\
\hline 40703-01-02-00 & 1999 Jan 08 01:40 & $3.85(1)$ & 0.111 & $\cdots$ & $\cdots$ & $9.9(6)$ \\
\hline 40703-01-03-00 & 1999 Jan 15 23:45 & $2.80(1)$ & 0.139 & $21(4)$ & $30(6)$ & $18.8(6)$ \\
\hline 40403-01-01-00 & 1999 Jan 24 22:04 & $2.98(1)$ & 0.129 & $17(4)$ & $14(6)$ & $15.2(8)$ \\
\hline 40703-01-04-02 & 1999 Feb 06 08:09 & $2.88(1)$ & 0.100 & $12(4)$ & $14(6)$ & $\ldots$ \\
\hline 40703-01-05-00 & 1999 Feb 12 01:12 & $3.71(1)$ & 0.101 & $68(4)$ & $65(6)$ & $47.3(8)$ \\
\hline
\end{tabular}

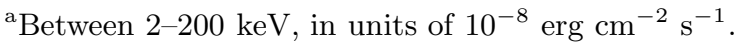

${ }^{\mathrm{b}}$ Ratio of PCA counts from 12-60 : 2-12 keV.

${ }^{\mathrm{c}}$ Observations which were not analyzed either because they were not taken with the necessary data modes or were too short for meaningful timing analyses. 
TABLE 2

Radio Observations of GRS 1915+105 while FAint

\begin{tabular}{|c|c|c|c|c|c|}
\hline Date & $\begin{array}{l}\text { Time } \\
(\mathrm{UT})\end{array}$ & Instrument & $\begin{array}{c}\text { Frequency } \\
\text { GHz }\end{array}$ & $\begin{array}{c}S_{\nu} \\
\text { mJy }\end{array}$ & $\begin{array}{c}\text { Beam } \\
(\operatorname{arcsec})\end{array}$ \\
\hline 1996 Oct 29 & $01: 15-02: 56$ & VLA & 8.4 & $30-70$ & $0.26 \times 0.23$ \\
\hline 1996 Oct 29 & $16: 37-19: 56$ & Ryle & 15.5 & 6.7 & 30 \\
\hline 1996 Nov 14 & $23: 37-01: 21$ & VLA & 8.4 & $18-8$ & $0.27 \times 0.24$ \\
\hline 1996 Nov 14 & $13: 34-18: 38$ & Ryle & 15.5 & 4.3 & 30 \\
\hline 1996 Dec 28 & $20: 50-23: 00$ & VLA & 8.4 & $4(1)$ & $0.32 \times 0.26$ \\
\hline 1996 Dec 28 & $20: 50-23: 00$ & GBI & 2.25 & $17(4)$ & 11 \\
\hline 1996 Dec 28 & $20: 50-23: 00$ & GBI & 8.3 & $14(6)$ & 3 \\
\hline 1996 Dec 28 & $09: 30-12: 30$ & Ryle & 15.2 & $6.4(4)$ & 30 \\
\hline 1997 Jan 12 & $21: 50-22: 30$ & VLA & 4.8 & $35-26$ & $0.63 \times 0.36$ \\
\hline 1997 Jan 12 & $21: 50-22: 30$ & VLA & 8.4 & $30-19$ & $0.41 \times 0.29$ \\
\hline 1997 Jan 12 & $12: 45-20: 57$ & GBI & 2.25 & $44(4)$ & 11 \\
\hline 1997 Jan 12 & $12: 45-20: 57$ & GBI & 8.3 & $21(6)$ & 3 \\
\hline 1997 Jan 13 & $16: 50-18: 35$ & VLA & 8.4 & $4.5(1)$ & $0.27 \times 0.24$ \\
\hline 1997 Jan 13 & $16: 50-18: 35$ & GBI & 2.25 & $14(4)$ & 11 \\
\hline 1997 Jan 13 & $16: 50-18: 35$ & GBI & 8.3 & $4(6)$ & 3 \\
\hline 1997 Apr 19 & $10: 40-15: 20$ & VLA & 1.4 & $3.9(1)$ & $4.5 \times 3.5$ \\
\hline 1997 Apr 19 & $10: 40-15: 20$ & GBI & 2.25 & $6(4)$ & 11 \\
\hline 1997 Apr 19 & $10: 40-15: 20$ & GBI & 8.3 & $6(6)$ & 3 \\
\hline 1998 Sep 14 & $04: 20-05: 30$ & VLA & 8.4 & $8.2(1)$ & $0.74 \times 0.70$ \\
\hline 1998 Sep 13 & $23: 07-04: 26$ & GBI & 2.25 & $18(4)$ & 11 \\
\hline 1998 Sep 13 & $23: 07-04: 26$ & GBI & 8.3 & $11(6)$ & 3 \\
\hline 1998 Sep 13 & $21: 39-22: 39$ & Ryle & 15.2 & $6.0(6)$ & 30 \\
\hline 1998 Sep 29 & $04: 00-06: 30$ & VLA & 5.0 & $12.2(1)$ & $2.26 \times 1.2$ \\
\hline 1998 Sep 29 & $04: 00-06: 30$ & VLA & 15.2 & $13.9(1)$ & $0.40 \times 0.38$ \\
\hline 1998 Sep 28 & $21: 36-03: 36$ & GBI & 2.25 & $20(4)$ & 11 \\
\hline 1998 Sep 28 & $21: 36-03: 36$ & GBI & 8.3 & $12(6)$ & 3 \\
\hline 1998 Sep 28 & $19: 07-21: 30$ & Ryle & 15.2 & $10.7(4)$ & 30 \\
\hline 1998 Oct 08 & $03: 30-05: 30$ & VLA & 5.0 & $31.7(1)$ & $1.6 \times 1.3$ \\
\hline 1998 Oct 08 & $03: 30-05: 30$ & VLA & 15.2 & $18.5(1)$ & $0.42 \times 0.41$ \\
\hline 1998 Oct 07 & $21: 22-03: 07$ & GBI & 2.25 & $60(4)$ & 11 \\
\hline 1998 Oct 07 & $21: 22-03: 07$ & GBI & 8.3 & $23(6)$ & 3 \\
\hline 1998 Oct 07 & $19: 05-21: 19$ & Ryle & 15.2 & $21.3(4)$ & 30 \\
\hline
\end{tabular}

